\title{
Gap probability at the hard edge for random matrix ensembles with pole singularities in the potential
}

\author{
Dan Dai, Shuai-Xia Xu ${ }^{\dagger}$ and Lun Zhang ${ }^{\ddagger}$
}

May 25, 2022

\begin{abstract}
We study the Fredholm determinant of an integrable operator acting on the interval $(0, s)$ whose kernel is constructed out of the $\Psi$-function associated with a hierarchy of higher order analogues to the Painlevé III equation. This Fredholm determinant describes the critical behavior of the eigenvalue gap probability at the hard edge of unitary invariant random matrix ensembles perturbed by poles of order $k$ in a certain scaling regime. Using the Riemann-Hilbert method, we obtain the large $s$ asymptotics of the Fredholm determinant. Moreover, we derive a Painlevé type formula of the Fredholm determinant, which is expressed in terms of an explicit integral involving a solution to a coupled Painlevé III system.
\end{abstract}

2010 Mathematics Subject Classification: 33E17; 34M55; 41A60.

Keywords: unitary ensembles; singular potentials; gap probability; asymptotics; Painlevé and coupled Painlevé equations; Riemann-Hilbert problem; Deift-Zhou steepest descent analysis.

\section{Contents}

1 Introduction

1.1 Random matrix ensembles with pole singularities in the potential . . . . . . . . 2

1.2 Double scaling limit of the correlation kernel at the hard edge . . . . . . . . . . 3

1.3 Connection with a Painlevé III hierarchy . . . . . . . . . . . . . . 6

1.4 Gap probability at the hard edge . . . . . . . . . . . . .

2 Statement of results 8

2.1 Large gap asymptotics . . . . . . . . . . . . . . . . . . . . . 8

2.2 A coupled Painlevé III system . . . . . . . . . . . . . . . . . . . . . . 9

2.3 A Painlevé type formula of the gap probability . . . . . . . . . . . 10

2.4 Organization of the rest of the paper . . . . . . . . . . . . . 10

\footnotetext{
* Department of Mathematics, City University of Hong Kong, Tat Chee Avenue, Kowloon, Hong Kong. E-mail: dandai@cityu.edu.hk

${ }^{\dagger}$ Institut Franco-Chinois de l’Energie Nucléaire, Sun Yat-sen University, Guangzhou 510275, China. E-mail: xushx3@mail.sysu.edu.cn

${ }^{\ddagger}$ School of Mathematical Sciences and Shanghai Key Laboratory for Contemporary Applied Mathematics, Fudan University, Shanghai 200433, China. E-mail: lunzhang@fudan.edu.cn
} 
3 Large $s$ asymptotics of $\frac{\partial}{\partial s} F(s ; \lambda)$

3.1 A Riemann-Hilbert setting . . . . . . . . . . . . . . . . . . 11

3.2 Asymptotic analysis of the Riemann-Hilbert problem for $X \ldots \ldots \ldots$. . . . 14

$3.2 .1 \quad X \rightarrow T$ : Rescaling . . . . . . . . . . . . . . . 14

$3.2 .2 T \rightarrow S:$ Normalization at $\infty$ and $0 \ldots \ldots \ldots \ldots$

3.2 .3 Outer parametrix . . . . . . . . . . . . . . . . . 17

3.2 .4 Local parametrix near $-1 \ldots \ldots \ldots \ldots \ldots \ldots$

3.2 .5 Final transformation . . . . . . . . . . . . . . . . 21

3.3 Large $s$ asymptotics of $\frac{\partial}{\partial s} F(s ; \lambda) \ldots \ldots \ldots \ldots \ldots \ldots$

4 Large $s$ asymptotics of $\frac{\partial}{\partial \lambda} F(s ; \lambda)$

4.1 A Riemann-Hilbert setting for $\frac{\partial}{\partial \lambda} F\left(\lambda^{2} s ; \lambda^{2}\right) \ldots \ldots \ldots \ldots \ldots . \ldots \ldots$

4.2 Asymptotic analysis of the Riemann-Hilbert problem for $\widehat{X} \ldots \ldots \ldots$. . . . 28

4.2.1 Transformations $\widehat{X} \rightarrow \widehat{T} \rightarrow \widehat{S} \ldots \ldots \ldots \ldots \ldots$

4.2.2 Outer parametrix, local parametrix near -1 and final transformation . . . 30

4.3 Large $s$ asymptotics of $\frac{\partial}{\partial \lambda} F(s ; \lambda) \ldots \ldots \ldots \ldots \ldots \ldots$

5 Proof of Theorem 2.1

6 Derivation of the coupled Painlevé III system 33

6.1 Riemann-Hilbert problem for $M \ldots \ldots \ldots \ldots \ldots \ldots$

6.2 Lax pair equations and a coupled Painlevé III system . . . . . . . . . . . . 35

7 Asymptotic analysis of the Riemann-Hilbert problem for $M$

$7.1 \quad M \rightarrow U$ : Rescaling and normalization $\ldots \ldots \ldots \ldots \ldots \ldots \ldots \ldots$

7.2 Outer parametrix . . . . . . . . . . . . . . . . . . . 38

7.3 Local parametrix near the origin . . . . . . . . . . . . . 39

7.4 Final transformation . . . . . . . . . . . . . . . . . . 41

8 Proofs of Theorems 2.4 and 2.5

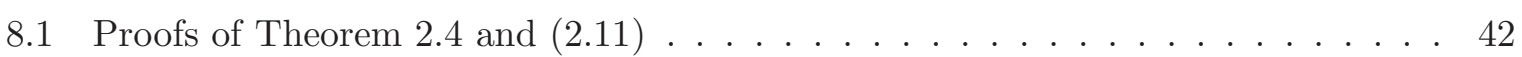

8.2 Proof of Theorem $2.5 \ldots \ldots \ldots \ldots \ldots \ldots \ldots$

A Consistency of (2.12) with the logarithm of (1.26) 44

\section{Introduction}

\subsection{Random matrix ensembles with pole singularities in the potential}

In this paper, we are concerned with the following unitary invariant random matrix ensembles

$$
\frac{1}{Z_{n}}(\operatorname{det} M)^{\alpha} \exp \left[-n \operatorname{tr} V_{k}(M)\right] d M, \quad \alpha>-1,
$$

defined on the space $\mathcal{H}_{n}^{+}$of $n \times n$ positive definite Hermitian matrices $M=\left(M_{i j}\right)_{1 \leq i, j \leq n}$, where

$$
\begin{aligned}
& d M=\prod_{i=1}^{n} d M_{i i} \prod_{1 \leq i<j \leq n} d \operatorname{Re} M_{i j} d \operatorname{Im} M_{i j}, \\
& Z_{n}=\int_{\mathcal{H}_{n}^{+}}(\operatorname{det} M)^{\alpha} \exp \left[-n \operatorname{tr} V_{k}(M)\right] d M
\end{aligned}
$$


is the normalization constant, and the potential

$$
V_{k}(x):=V(x)+\left(\frac{t}{x}\right)^{k}, \quad x \in(0, \infty), \quad t>0, \quad k \in \mathbb{N} .
$$

In (1.4), it is assumed that the regular part $V$ of the potential is real analytic on $[0, \infty)$, and also we are in the one-cut regular case in the sense of [21, 30]. This particularly implies that the limiting mean distribution of the eigenvalues as $n \rightarrow \infty$ for $t=0$ is supported on $[0, b]$ for some positive $b$.

Since the ensembles are unitary invariant, we have that the eigenvalues of a random matrix in (1.1) form a determinantal point process [29, 34, whose joint probability density function is explicitly given by

$$
\frac{1}{\widehat{Z}_{n}} \prod_{1 \leq i<j \leq n}\left(x_{j}-x_{i}\right)^{2} \prod_{j=1}^{n} w\left(x_{j}\right)
$$

with

$$
\widehat{Z}_{n}=\int_{[0, \infty)^{n}} \prod_{1 \leq i<j \leq n}\left(x_{j}-x_{i}\right)^{2} \prod_{j=1}^{n} w\left(x_{j}\right) d x_{j}
$$

and

$$
w(x)=x^{\alpha} e^{-n V_{k}(x)} .
$$

Clearly, random matrix ensembles of the form (1.1) can be interpreted as unitary ensembles perturbed by a pole of order $k$ at the origin. These singularly perturbed ensembles have attracted lots of interests recently. On one hand, they arise in a variety of problems. These problems include statistics for zeta zeros and eigenvalues [4, eigenvalues of Wigner-Smith timedelay matrix in the context of quantum transport and electrical characteristics of chaotic cavities [10, 11, 36], and random matrix models in the field of spin-glasses [2], etc.. On the other hand, these ensembles are natural candidates to exhibit new critical phenomena. Indeed, for each fixed $t>0$, the eigenvalues are repelled from the origin due to the pole singularities in the potential. This behavior is quite different from the unperturbed case (i.e., $t=0$ ), in which the eigenvalues will accumulate near the origin as $n \rightarrow \infty$ for the one-cut regular case. As a consequence, it is expected that some new phenomena will appear near the origin if one takes $t \rightarrow 0$ and simultaneously $n \rightarrow \infty$, which also corresponds to a phase transition between two different edge behaviors. Studies of this aspect have been conducted in [9] for the perturbed Gaussian unitary ensembles, in [14, 32, 43, 44] for the perturbed Laguerre unitary ensembles, and in $[3$ for the general case as indicated in (1.1). It comes out that the Painlevé III equation and its hierarchy play an important role in describing the critical behavior, as we will review from the viewpoint of correlation kernel in what follows.

\subsection{Double scaling limit of the correlation kernel at the hard edge}

Let us denote by $K_{n}(x, y ; t)$ the correlation kernel associated with (1.5), which takes the following form

$$
K_{n}(x, y ; t)=h_{n-1}^{-1} \sqrt{w(x) w(y)} \frac{\pi_{n}(x) \pi_{n-1}(y)-\pi_{n-1}(x) \pi_{n}(y)}{x-y}, \quad x, y>0 .
$$

Here $\pi_{j}(x), j=0,1, \cdots$, is a family of monic polynomials of degree $j$ satisfying the orthogonality conditions

$$
\int_{0}^{\infty} \pi_{j}(x) \pi_{m}(x) w(x) d x=h_{j} \delta_{j, m},
$$




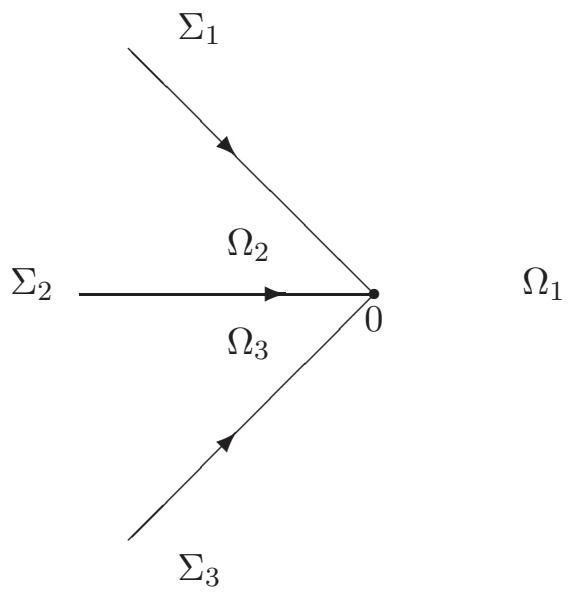

Figure 1: The jump contours $\Sigma_{j}$ and the regions $\Omega_{j}, j=1,2,3$, for the $\mathrm{RH}$ problem for $\Psi$.

where the weight function $w$ is given in (1.6).

To state the relevant results, we also need the following Riemann-Hilbert( $\mathrm{RH})$ problem.

RH problem 1.1. Let $\lambda>0$ and $\alpha>-1$. We look for a $2 \times 2$ matrix-valued function $\Psi(z)=\Psi(z ; \lambda)$ satisfying

(1) $\Psi(z)$ is defined and analytic in $\mathbb{C} \backslash\left\{\cup_{j=1}^{3} \Sigma_{j} \cup\{0\}\right\}$, where the contours $\Sigma_{j}, j=1,2,3$, are illustrated in Figure 1

(2) $\Psi$ has limiting values $\Psi_{ \pm}(z)$ for $z \in \cup_{j=1}^{3} \Sigma_{j}$, where $\Psi_{+}\left(\Psi_{-}\right)$denotes the limiting values from the left (right) side of $\Sigma_{j}$, and

$$
\Psi_{+}(z)=\Psi_{-}(z) \begin{cases}\left(\begin{array}{cc}
1 & 0 \\
e^{\alpha \pi i} & 1
\end{array}\right), & z \in \Sigma_{1}, \\
\left(\begin{array}{cc}
0 & 1 \\
-1 & 0
\end{array}\right), & z \in \Sigma_{2}, \\
\left(\begin{array}{cc}
1 & 0 \\
e^{-\alpha \pi i} & 1
\end{array}\right), & z \in \Sigma_{3} .\end{cases}
$$

(3) As $z \rightarrow \infty$, there exist functions $p(\lambda), q(\lambda)$ and $r(\lambda)$ such that

$$
\Psi(z ; \lambda)=\left(I+\frac{1}{z}\left(\begin{array}{cc}
q(\lambda) & -i r(\lambda) \\
i p(\lambda) & -q(\lambda)
\end{array}\right)+O\left(z^{-2}\right)\right) z^{-\frac{1}{4} \sigma_{3}} \frac{I+i \sigma_{1}}{\sqrt{2}} e^{z^{\frac{1}{2}} \sigma_{3}},
$$

where the branch cuts of $z^{-\frac{1}{4}}$ and $z^{\frac{1}{2}}$ are taken along the negative real axis, and

$$
\sigma_{1}=\left(\begin{array}{cc}
0 & 1 \\
1 & 0
\end{array}\right), \quad \sigma_{3}=\left(\begin{array}{cc}
1 & 0 \\
0 & -1
\end{array}\right)
$$

are the Pauli matrices. 
(4) As $z \rightarrow 0$, there exists a unimodular matrix $\Psi_{0}(\lambda)$, independent of $z$, such that

$$
\Psi(z ; \lambda)=\Psi_{0}(\lambda)(I+O(z)) e^{-\left(-\frac{\lambda}{z}\right)^{k} \sigma_{3}} z^{\frac{\alpha}{2} \sigma_{3}}\left\{\begin{array}{cc}
I, & \\
\left(\begin{array}{rr}
1 & 0 \\
-e^{\alpha \pi i} & 1
\end{array}\right), & z \in \Omega_{2}, \\
\left(\begin{array}{cc}
1 & 0 \\
e^{-\alpha \pi i} & 1
\end{array}\right), & z \in \Omega_{3},
\end{array}\right.
$$

where the regions $\Omega_{i}, i=1,2,3$, are depicted in Figure 1.

It was shown in [3] that there exists a unique solution to the above $\mathrm{RH}$ problem for each $k \in \mathbb{N}, \alpha>-1$ and $\lambda>0$. This generalizes the result in [44] for the case $k=1$.

Now, for $z \in \Omega_{1}$, let us set

$$
\left(\begin{array}{l}
\psi_{1}(z ; \lambda) \\
\psi_{2}(z ; \lambda)
\end{array}\right):=\Psi(z ; \lambda)\left(\begin{array}{l}
1 \\
0
\end{array}\right) .
$$

These two functions can be extended analytically in the whole complex plane with a cut along $\Sigma_{1}$, which we still denote by $\psi_{i}(z ; \lambda), i=1,2$. This particularly implies that

$$
\left(\begin{array}{l}
\psi_{1}(x ; \lambda) \\
\psi_{2}(x ; \lambda)
\end{array}\right)=\Psi_{-}(x ; \lambda)\left(\begin{array}{c}
1 \\
-e^{-\alpha \pi i}
\end{array}\right), \quad x<0 .
$$

Let us consider the correlation kernel $K_{n}(x, y ; t)$ given in (1.7). Assume that the parameter $t \rightarrow 0$ and $n \rightarrow \infty$ in such a way that

$$
2^{-\frac{1}{k}} c_{V} n^{\frac{2 k+1}{k}} t \rightarrow \lambda>0
$$

where $c_{V}$ is a constant depending on the regular part $V$ in the potential. It was shown in $[3$, Theorem 1.10] that

$$
\lim _{n \rightarrow \infty} \frac{1}{c_{V} n^{2}} K_{n}\left(\frac{u}{c_{V} n^{2}}, \frac{v}{c_{V} n^{2}} ; t\right)=K_{\mathrm{PIII}}(u, v ; \lambda),
$$

uniformly for $u, v$ and $\lambda$ in any compact subsets of $(0, \infty)$, where

$$
K_{\mathrm{PIII}}(u, v ; \lambda):=e^{\alpha \pi i} \frac{\psi_{1}(-u ; \lambda) \psi_{2}(-v ; \lambda)-\psi_{1}(-v ; \lambda) \psi_{2}(-u ; \lambda)}{2 \pi i(u-v)} .
$$

The subscript PIII is used to indicate that the RH problem 1.1 for $\Psi$ is related to a hierarchy of higher order analogues to the Painlevé III equation, which we will explain next.

Remark 1.2. When $\lambda=0$, RH problem 1.1 for $\Psi$ can be solved explicitly in terms of modified Bessel functions; cf. [31. Furthermore, this also corresponds to the case $t=0$ in (1.4), i.e., the singular part in the potential $V_{k}$ vanishes. Since the hard edge scaling limit of the correlation kernel for the unperturbed unitary ensembles is given by the classical Bessel kernel [25] in general (cf. [41]), it is then natural to expect some transition will occur between these two kernels as the parameter $\lambda$ varies. This is indeed the case. According to [3, 44, we have

$$
K_{\mathrm{PIII}}(u, v ; \lambda)=K_{\mathrm{Bes}}(u, v)+O(\lambda), \quad \lambda \rightarrow 0^{+},
$$


uniformly for $u, v$ in any compact subset of $(0, \infty)$, where

$$
K_{\mathrm{Bes}}(x, y)=\frac{J_{\alpha}(\sqrt{x}) \sqrt{y} J_{\alpha}^{\prime}(\sqrt{y})-\sqrt{x} J_{\alpha}^{\prime}(\sqrt{x}) J_{\alpha}(\sqrt{y})}{2(x-y)},
$$

is the Bessel kernel of order $\alpha$, and $J_{\alpha}(x)$ is the Bessel function of the first kind of order $\alpha$; see [1].

\subsection{Connection with a Painlevé III hierarchy}

The $(1,2)$ entry $r(\lambda)$ of the residue term at infinity for $\Psi$ in (1.10) is connected to a Painlevé III hierarchy. More precisely, given $k \in \mathbb{N}$, let us consider the following $k+1$ ODEs for $k+1$ unknown functions $\left(\varrho(\lambda), \ell_{1}(\lambda), \ldots, \ell_{k}(\lambda)\right)$ indexed by $p=0, \ldots, k$ :

$$
\begin{cases}\varrho=-\frac{1}{4 \ell_{k}^{2}}\left(\left(\ell_{k}^{2}\right)^{\prime \prime}-3\left(\ell_{k}^{\prime}\right)^{2}+\tau_{0}\right), & p=0, \\ \sum_{q=0}^{p}\left(\ell_{k-p+q+1} \ell_{k-q}-\left(\ell_{k-p+q} \ell_{k-q}\right)^{\prime \prime}+3 \ell_{k-p+q}^{\prime} \ell_{k-q}^{\prime}-4 \varrho \ell_{k-p+q} \ell_{k-q}\right)=\tau_{p}, & 1 \leq p \leq k,\end{cases}
$$

where $\left({ }^{\prime}\right)=\frac{d}{d \lambda}, \tau_{p}, p=0,1, \ldots, k$ are real constants, and

$$
\ell_{k+1}(\lambda)=0, \quad \ell_{0}(\lambda)=\frac{\lambda}{2} .
$$

If $k=1$, the ODE for $\ell_{1}$ reads

$$
\ell_{1}^{\prime \prime}(\lambda)=\frac{\ell_{1}^{\prime}(\lambda)^{2}}{\ell_{1}(\lambda)}-\frac{\ell_{1}^{\prime}(\lambda)}{\lambda}-\frac{\ell_{1}(\lambda)^{2}}{\lambda}-\frac{\tau_{0}}{\ell_{1}(\lambda)}+\frac{\tau_{1}}{\lambda},
$$

which can be identified as the Painlevé III equation [33, Equation (32.2.3)] with parameters $\alpha=-1, \beta=\tau_{1}, \gamma=0$ and $\delta=-\tau_{0} p$. Hence, the system of equations (1.19) is called the $k$-th member of the Painlevé III hierarchy. For general $k>1$, one can obtain an ODE of order $2 k$ for $\ell_{1}$ from (1.19) by eliminating the other functions $\varrho$ and $\ell_{p}(2 \leq p \leq k)$.

The connection between $r(\lambda)$ and the Painlevé III hierarchy (1.19) is shown in [3, Theorem 1.6], as stated in the following proposition.

Proposition 1.3. Let $\Psi(z ; \lambda)$ be the unique solution to $R H$ problem 1.1 for $\alpha>-1$ and $\lambda>0$. Then, the limit

$$
y_{\alpha}(\lambda)=-2 i \frac{d}{d \lambda}\left(\lim _{z \rightarrow \infty} z \Psi\left(z ; \lambda^{2}\right) e^{-z^{\frac{1}{2}} \sigma_{3}} \frac{I-i \sigma_{1}}{\sqrt{2}} z^{\frac{1}{4} \sigma_{3}}\right)_{12}=-2 \frac{d}{d \lambda}\left(r\left(\lambda^{2}\right)\right)
$$

exists, where $(M)_{i j}$ stands for the $(i, j)$-th entry of a matrix $M$. Furthermore, $y_{\alpha}(\lambda)$ is a solution of the equation for $\ell_{1}$ of the $k$-th member of the Painleve III hierarchy (1.19), with the parameters $\tau_{p}, p=0, \ldots, k$ given by

$$
\tau_{p}= \begin{cases}4^{2 k+1} k^{2}, & p=0, \\ -(-4)^{k+1} \alpha k, & p=k, \\ 0, & 0<p<k .\end{cases}
$$

In addition, the asymptotic behavior of $y_{\alpha}$ can be derived from the following asymptotics of $r(\lambda)$ :

$$
r(\lambda)=\frac{1}{8}\left(1-4 \alpha^{2}\right)+O\left(\lambda^{k}\right), \quad \text { as } \lambda \rightarrow 0^{+}
$$


and

$$
r(\lambda)=\lambda^{\frac{2 k}{2 k+1}}\left(\beta_{k-2}-\frac{3}{2} z_{0}\right)-\left(-z_{0}\right)^{\frac{1}{2}} \lambda^{\frac{k}{2 k+1}} \alpha+O(1), \quad \text { as } \lambda \rightarrow+\infty
$$

where

$$
z_{0}=-\left(\frac{2^{k-1}(k-1) !}{(2 k-1) ! !}\right)^{-\frac{2}{2 k+1}}, \quad \beta_{j}=-\left(-z_{0}\right)^{-\frac{3}{2}-j} \frac{(2 j+1) ! !}{2^{j} j !}
$$

with $(2 j+1) ! !=(2 j+1)(2 j-1) \cdots 3 \cdot 1$ being the double factorial; see [3, Equations $(5.17)$ and $(4.57)]$.

\subsection{Gap probability at the hard edge}

Let $\mathcal{K}_{\text {PIII }}$ be the integral operator with kernel $\chi_{[0, s]}(u) K_{\text {PIII }}(u, v) \chi_{[0, s]}(v)$ in (1.16) acting on the function space $L^{2}((0, \infty))$, where $s>0$ and $\chi_{J}$ is the characteristic function of the interval $J$. It is the aim of this paper to study the Fredholm determinant

$$
\operatorname{det}\left(I-\mathcal{K}_{\mathrm{PIII}}\right) \text {. }
$$

Due to the determinantal structure (1.5), the above function gives us the gap probability (the probability of finding no eigenvalues) on the interval $(0, s)$ for the limiting process of the random matrix ensembles (1.1), i.e.,

$$
\begin{aligned}
& \operatorname{det}\left(I-\mathcal{K}_{\text {PIII }}\right) \\
& \quad=\lim \operatorname{Prob}\left(M \text { distributed according to (1.1) has no eigenvalues in }\left(0, \frac{s}{c_{V} n^{2}}\right)\right),
\end{aligned}
$$

where the limit is understood that both $t \rightarrow 0$ and $n \rightarrow \infty$ such that condition (1.14) holds.

It is well-known that, for many limiting correlation kernels arising from random matrix theory, the associated Fredholm determinants are related to systems of integrable differential equations [37]-[40]. Note that kernel $K_{\mathrm{PIII}}(u, v)$ in (1.16) can be viewed as a generalization of the classical Bessel kernel $K_{\text {Bes }}(u, v)$ in (1.18) (see Remark 1.2). Let us recall some basic facts regarding the Bessel kernel.

Define $\mathcal{K}_{\text {Bes }}$ to be the integral operator with kernel $\chi_{[0, s]}(u) K_{\text {Bes }}(u, v) \chi_{[0, s]}(v)$ acting on the function space $L^{2}((0, \infty))$. The celebrated Tracy-Widom formula (see [38, Equation (1.19)]) is

$$
\operatorname{det}\left(I-\mathcal{K}_{\mathrm{Bes}}\right)=\exp \left(-\frac{1}{4} \int_{0}^{s} \log \left(\frac{s}{\tau}\right) \mathfrak{q}^{2}(\tau) d \tau\right)
$$

where the function $\mathfrak{q}(\tau)$ satisfies the following equation

$$
\tau\left(\mathfrak{q}^{2}-1\right)\left(\tau \mathfrak{q}^{\prime}\right)^{\prime}=\mathfrak{q}\left(\tau \mathfrak{q}^{\prime}\right)^{2}+\frac{1}{4}\left(\tau-\alpha^{2}\right) \mathfrak{q}+\frac{1}{4} \tau \mathfrak{q}^{3}\left(\mathfrak{q}^{2}-2\right)
$$

with the boundary condition

$$
\mathfrak{q}(\tau) \sim \frac{1}{2^{\alpha} \Gamma(1+\alpha)} \tau^{\alpha / 2}, \quad \text { as } \tau \rightarrow 0^{+} .
$$

Note that, the equation (1.27) reduces to a special case of the Painlevé $\mathrm{V}$ equation via the transformation $y(\tau)=\left(q\left(\tau^{2}\right)-1\right) /\left(q\left(\tau^{2}\right)+1\right)$. Furthermore, the following large $s$ asymptotics of $\operatorname{det}\left(I-\mathcal{K}_{\mathrm{Bes}}\right)$ is conjectured in [38], and later rigorously proved in [20] (see also [6]):

$$
\ln \operatorname{det}\left(I-\mathcal{K}_{\mathrm{Bes}}\right)=-\frac{1}{4} s+\alpha s^{1 / 2}-\frac{\alpha^{2}}{4} \ln s+\tau_{\alpha}+O\left(s^{-1 / 2}\right), \quad \text { as } s \rightarrow+\infty,
$$


where the constant $\tau_{\alpha}$ is given by

$$
\tau_{\alpha}=\ln \left(\frac{G(1+\alpha)}{(2 \pi)^{\alpha / 2}}\right)
$$

with $G(z)$ being the Barnes $G$-function.

It would then be natural to derive the large $s$ asymptotics of $\operatorname{det}\left(I-\mathcal{K}_{\mathrm{PIII}}\right)$ and to ask for its Painlevé type formula, which will be the main results of the present work stated in what follows. We note that similar problems have been addressed for other generalizations of Bessel kernel recently in [16, 26, 35, 45].

\section{Statement of results}

\subsection{Large gap asymptotics}

Our first result is the following large $s$ asymptotics of the gap probability.

Theorem 2.1. For $\alpha>-1, s>0$ and $\lambda>0$, let $\mathcal{K}_{P I I I}$ be the integral operator with kernel $\chi_{[0, s]}(u) K_{P I I I}(u, v) \chi_{[0, s]}(v)$ in (1.16) acting on the function space $L^{2}((0, \infty))$ and denote

$$
F(s ; \lambda):=\ln \operatorname{det}\left(I-\mathcal{K}_{P I I I}\right) .
$$

Then, we have, as $s \rightarrow+\infty$,

$$
F(s ; \lambda)=-\frac{1}{4} s+\alpha s^{1 / 2}-\frac{\alpha^{2}}{4} \ln s+\int_{0}^{\lambda} \frac{1}{2 t}\left(r(t)+\frac{\alpha^{2}}{2}-\frac{1}{8}\right) d t+\tau_{\alpha}+O\left(s^{-1 / 2}\right),
$$

where the function $r(t)$ is related to the Painlevé III hierarchy as stated in Proposition 1.3 and the constant $\tau_{\alpha}$ is given in (1.30).

In view of the local behavior of $r(\lambda)$ near the origin given in (1.22), the integral in (2.2) is well-defined. Moreover, as $\lambda \rightarrow 0^{+}$, we recover the large $s$ asymptotics of $\ln \operatorname{det}\left(I-\mathcal{K}_{\text {Bes }}\right)$ shown in (1.29). This is compatible with the fact (1.17), and also explains why the two constant terms in (1.29) and (2.2) are the same.

Remark 2.2. In the literature, the asymptotics of Fredholm determinants of other Painlevé kernels have been investigated in [17] for the kernels built in terms of the Painlevé I hierarchy, in [7] for the Painlevé II kernel associated with the Hastings-McLeod solution, and quite recently in 42 for the Painlevé XXXIV kernel. All these Painlevé kernels describe certain critical behaviors encountered in random matrix theory.

Remark 2.3. We would like to point out that our asymptotic formula (2.2) provides another concrete example that supports the so-called Forrester-Chen-Eriksen-Tracy conjecture [13, 25]. This conjecture asserts that if the mean density $\rho(x)$ of particles around a point $x^{*}$ behaves like $\left(x-x^{*}\right)^{\beta}$, then, for the probability $E(s)$ of emptiness of the interval $\left(x^{*}-s, x^{*}+s\right)$, we have

$$
E(s) \sim \exp \left(-C s^{2 \beta+2}\right) .
$$

When $E(s)$ admits a representation in terms of a Fredholm determinant associated with certain kernel, this conjecture is supported by all the classical kernels encountered in random matrix theory, which include the sine kernel [23] $(\beta=0)$, the Airy kernel [39] $(\beta=1 / 2)$ and the Bessel kernel (1.29) $(\beta=-1 / 2)$. Other evidences beyond these classical cases include higher Painlevé I kernels [17] $(\beta=2 l+1 / 2, l \in \mathbb{N})$, the Painlevé II kernel [7] $(\beta=2)$, the 
Meijer G-kernels $\left(\beta=-\frac{l}{l+1}, l \in \mathbb{N}\right)$ and Wright's generalized Bessel kernels $\left(\beta=-\frac{1}{1+\theta}, \theta>0\right)$ investigated in [16].

For the model (1.1) considered in the present work, the limiting mean distribution of the eigenvalues as $n \rightarrow \infty$ and $t \rightarrow 0^{+}$is the same as the unperturbed case, which could be described as the minimizer of an equilibrium problem with external field $V$. Due to our assumption on the regularity of the potential $V$, the density function behaves like $x^{-1 / 2}$ near the origin. Hence, the asymptotic formula (2.2) indeed supports (2.3) with $\beta=-1 / 2$.

Finally, we highlight that the $s$-independent term in (2.2) involves an integral with respect to certain Painlevé transcendents. This phenomena was first observed in [7] in the studies of Painlevé II kernel associated with the Hastings-McLeod solution, and is also confirmed in recent work 42 concerning the Painlevé XXXIV kernel. We expect this feature is true for the large $s$ asymptotics associated with any other Painlevé kernels.

\subsection{A coupled Painlevé III system}

To express a Painlevé type formula for the gap probability, we need a function $a(\lambda ; s)$. This function, together with the other $k+1$ functions $b_{1}(\lambda ; s), \ldots, b_{k+1}(\lambda ; s)$, satisfies the following system of coupled ODEs involving $k+2$ equations:

$$
\left\{\begin{array}{l}
\left(\lambda-2 b_{1}\right) b_{1}^{\prime \prime}+\left(b_{1}^{\prime}-\frac{1}{2}\right)^{2}-\left(2 a^{\prime}-s\right)\left(\lambda-2 b_{1}\right)^{2}=0, \\
\sum_{m=j-k-1}^{k+1}\left(-b_{j-m} b_{m}^{\prime \prime}+\frac{b_{j-m}^{\prime} b_{m}^{\prime}}{2}+4 a^{\prime} b_{j-m} b_{m}+2 b_{j-m} b_{m+1}\right)=\Lambda_{j}, \quad k+2 \leq j \leq 2 k+2,
\end{array}\right.
$$

where $^{\prime}=\frac{d}{d \lambda}, b_{k+2}(\lambda ; s):=0$ and the constants $\Lambda_{j}$ are given by

$$
\Lambda_{j}= \begin{cases}2 k^{2}, & j=2 k+2, \\ (-1)^{k} 2 \alpha k, & j=k+2 \\ 0, & k+2<j<2 k+2\end{cases}
$$

In addition, we also have

$$
\frac{\partial}{\partial s} a(\lambda ; s)=\frac{\lambda}{2}-b_{1}(\lambda ; s) .
$$

We call the system (2.4) a coupled Painlevé III system for the following reason. When $k=1$, the ODEs in (2.4) reduce to

$$
\left\{\begin{array}{l}
\left(\lambda-2 b_{1}\right) b_{1}^{\prime \prime}+\left(b_{1}^{\prime}-\frac{1}{2}\right)^{2}-\left(2 a^{\prime}-s\right)\left(\lambda-2 b_{1}\right)^{2}=0, \\
-b_{2} b_{2}^{\prime \prime}+\frac{1}{2}\left(b_{2}^{\prime}\right)^{2}+4 a^{\prime} b_{2}^{2}=2, \\
b_{1} b_{2}^{\prime \prime}-b_{1}^{\prime \prime} b_{2}-\frac{b_{1}\left(b_{2}^{\prime}\right)^{2}}{b_{2}}+b_{1}^{\prime} b_{2}^{\prime}+2 b_{2}^{2}+2 \alpha+\frac{4 b_{1}}{b_{2}}=0 .
\end{array}\right.
$$

Set $b_{1}=\lambda / 2$, the first equation in (2.7) is satisfied automatically, while the third equation for $b_{2}$ now reads

$$
b_{2}^{\prime \prime}-\frac{\left(b_{2}^{\prime}\right)^{2}}{b_{2}}+\frac{1}{\lambda}\left(b_{2}^{\prime}+4 b_{2}^{2}+4 \alpha\right)+\frac{4}{b_{2}}=0,
$$

which is a Painlevé III equation.

For general $k \in \mathbb{N}$, if $s=0$, there exist solutions $a(\lambda)$ and $b_{i}(\lambda), i=1, \ldots, k+1$, to the system (2.4) with $b_{1}=\lambda / 2$ (see Remark 6.6 below). It is then straightforward to check that the ODEs in (2.4) are related to the Painlevé III hierarchy (1.19) through the following relations

$$
\left\{\begin{array}{l}
a^{\prime}(\lambda)=-\varrho(\lambda) / 2, \\
b_{j+1}(\lambda)=\ell_{j}(\lambda) / 4^{j}, \quad j=0, \cdots, k .
\end{array}\right.
$$


Our second result concerns the existence of a class of special solutions to the above coupled Painlevé III system.

Theorem 2.4. For $\alpha>-1, s>0$, there exist solutions $a(\lambda ; s)$ and $b_{1}(\lambda ; s), \ldots, b_{k+1}(\lambda ; s)$ to the coupled Painlevé III system (2.4), which are analytic for $\lambda \in(0,+\infty)$ and the function $a(\lambda ; s)$ satisfies the following asymptotic behaviors as $\lambda \rightarrow 0^{+}$:

$$
a(\lambda ; s)=\frac{1-4 \alpha^{2}}{8 \lambda}+O\left(\lambda^{1+2 \alpha}\right) .
$$

In principle, we could also derive the asymptotics of other functions $b_{i}(\lambda ; s), i=1, \ldots, k+1$, for the special solutions in Theorem 2.4. For instance, we have

$$
b_{1}(\lambda ; s)=\frac{\lambda}{2}-\frac{s^{\alpha} \lambda^{2 \alpha+1}}{2^{2 \alpha+1} \Gamma(\alpha+1)^{2}} e^{-\frac{2}{s^{k}}}\left(1+O\left(\lambda^{2(1+\alpha)}\right)\right), \quad \lambda \rightarrow 0^{+} .
$$

Since it is the function $a(\lambda ; s)$ that will play a role in our Painlevé type formula below, we will not discuss their asymptotics in this paper.

\subsection{A Painlevé type formula of the gap probability}

Our final result shows the existence of a Painlevé type formula of the gap probability, after proper scaling.

Theorem 2.5. With the logarithm of the gap probability $F(s ; \lambda)$ defined in (2.1), we have

$$
F\left(\lambda^{2} s ; \lambda^{2}\right)=-\int_{0}^{\lambda}[a(\tau ; s)-a(\tau ; 0)] d \tau
$$

where the function $a(\lambda ; s)$ is among the class of special solutions to the coupled Painlevé III system (2.4) as stated in Theorem 2.4.

By (2.10), it is readily seen that the integral in (2.12) is convergent. We also emphasize that the formula (2.12) is consistent with the Tracy-Widom formula for Bessel kernel in the sense that it boils down to the logarithm of (1.26) as $\lambda \rightarrow 0^{+}$. For convenience of the reader, we will discuss this aspect in the Appendix.

Remark 2.6. It is worthwhile to note that the relations between Fredholm determinants and other coupled Painlevé systems have also been established in recent studies, which generalize the classical results of Tracy and Widom [38, 39]. More precisely, the coupled Painlevé II systems have been related to the generating function for the Airy point process in [15, to the Fredholm determinants of the Painlevé II and the Painlevé XXXIV kernel in [42. A coupled Painlevé V system has been related to the generating function for the Bessel point process in [12].

\subsection{Organization of the rest of the paper}

The rest of this paper is devoted to the proofs of our results. Due to the integrable structure of the kernel $K_{\mathrm{PIII}}(u, v)$ in the sense of Its-Izergin-Korepin-Slavnov [27, the proof of Theorem 2.1 relies on the representations of partial derivatives of Fredholm determinant in terms of the solutions of certain RH problems, established under a general framework in [5, 19. In Section 3. we formulate a $\mathrm{RH}$ problem $X$ with constant jumps related to $\frac{\partial}{\partial s} F(s ; \lambda)$ and then perform a Deift-Zhou steepest descent analysis [22] on this RH problem, which ultimately leads to the large $s$ asymptotics of $\frac{\partial}{\partial s} F(s ; \lambda)$. Similarly, we study a RH problem $\widehat{X}$ associated with $\frac{\partial}{\partial \lambda} F\left(\lambda^{2} s ; \lambda^{2}\right)$ 
and its large $s$ asymptotics in Section 4 . The differences between the RH problems for $X$ and $\widehat{X}$ lie in the boundary conditions. This asymptotics, together with the asymptotics of $\frac{\partial}{\partial s} F(s ; \lambda)$, will lead to large $s$ asymptotics of $\frac{\partial}{\partial \lambda} F(s ; \lambda)$. A combination of these two asymptotics of partial derivatives of $F(s ; \lambda)$ gives us the proof of Theorem 2.1, as shown in Section 5. The derivation of the coupled Painlevé III system is given in Section 6, where we formulate a model RH problem $M$ and the coupled Painlevé III system follows from the compatibility conditions of the Lax pair equations associated with this $\mathrm{RH}$ problem. The $\mathrm{RH}$ problem for $M$ is actually defined as a 'scaled' version of that for $X$, and is also related to $\frac{\partial}{\partial s} F\left(\lambda^{2} s ; \lambda^{2}\right)$. After performing an asymptotic analysis of this RH problem in Section 7, we present the proofs of Theorems 2.4 and 2.5 in Section 8 . Finally, the consistency of (2.12) with the logarithm of (1.26) is discussed in the Appendix.

\section{$3 \quad$ Large $s$ asymptotics of $\frac{\partial}{\partial s} F(s ; \lambda)$}

In this section, we will derive large $s$ asymptotics of $\frac{\partial}{\partial s} F(s ; \lambda)$ by relating $\frac{\partial}{\partial s} F(s ; \lambda)$ to a $\mathrm{RH}$ problem. The strategy follows from a general framework established in [5, 19]. We first recall some basic facts in the context of our model.

\subsection{A Riemann-Hilbert setting}

To facilitate our computations, we shall make use of the fact that

$$
\operatorname{det}\left(I-\mathcal{K}_{\mathrm{PIII}}\right)=\operatorname{det}\left(I-\widetilde{\mathcal{K}}_{\mathrm{PIII}}\right),
$$

where $\widetilde{\mathcal{K}}_{\text {PIII }}$ is the integral operator with kernel

$$
\chi_{[-s, 0]}(u) \widetilde{K}_{\mathrm{PIII}}(u, v) \chi_{[-s, 0]}(v)=e^{\alpha \pi i} \chi_{[-s, 0]}(u) \frac{\psi_{1}(v ; \lambda) \psi_{2}(u ; \lambda)-\psi_{1}(u ; \lambda) \psi_{2}(v ; \lambda)}{2 \pi i(u-v)} \chi_{[-s, 0]}(v)
$$

acting on the function space $L^{2}((-\infty, 0))$.

By (3.2), it is readily seen that

$$
\widetilde{K}_{\mathrm{PIII}}(u, v)=\frac{\mathbf{f}^{t}(u) \mathbf{h}(v)}{u-v}
$$

where

$$
\mathbf{f}=-e^{\frac{\alpha \pi i}{2}}\left(\begin{array}{c}
\psi_{1} \\
\psi_{2}
\end{array}\right), \quad \mathbf{h}=\frac{e^{\frac{\alpha \pi i}{2}}}{2 \pi i}\left(\begin{array}{c}
\psi_{2} \\
-\psi_{1}
\end{array}\right)
$$

In addition, we have

$$
\frac{d}{d s} \ln \operatorname{det}\left(I-\widetilde{\mathcal{K}}_{\mathrm{PIII}}\right)=-\operatorname{tr}\left(\left(I-\widetilde{\mathcal{K}}_{\mathrm{PIII}}\right)^{-1} \frac{d}{d s} \widetilde{\mathcal{K}}_{\mathrm{PIII}}\right)=-R(-s,-s),
$$

where $R(u, v)$ stands for the kernel of the resolvent operator

$$
R=\left(I-\widetilde{\mathcal{K}}_{\text {PIII }}\right)^{-1}-I=\widetilde{\mathcal{K}}_{\text {PIII }}\left(I-\widetilde{\mathcal{K}}_{\text {PIII }}\right)^{-1}=\left(I-\widetilde{\mathcal{K}}_{\text {PIII }}\right)^{-1} \widetilde{\mathcal{K}}_{\text {PIII }} .
$$

Since the operator $\widetilde{\mathcal{K}}_{\text {PIII }}$ is integrable, its resolvent kernel is integrable as well; cf. [19, 27]. Indeed, by setting

$$
\mathbf{F}:=\left(I-\widetilde{\mathcal{K}}_{\text {PIII }}\right)^{-1} \mathbf{f}, \quad \mathbf{H}:=\left(I-\widetilde{\mathcal{K}}_{\text {PIII }}\right)^{-1} \mathbf{h},
$$




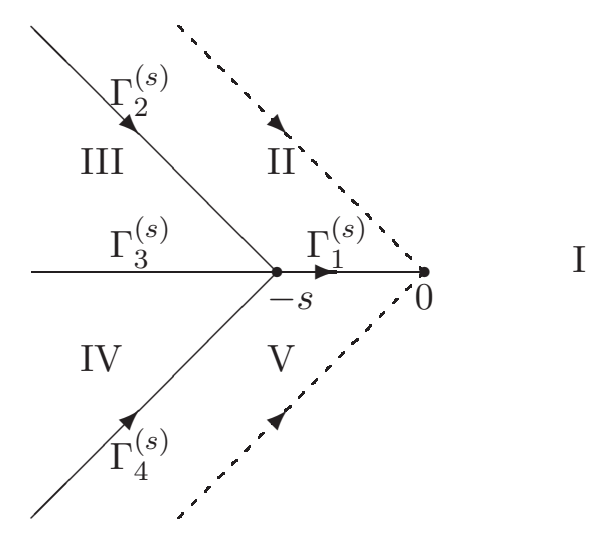

Figure 2: Regions I - V and contours $\Gamma_{j}^{(s)}, j=1,2,3,4$.

we have

$$
R(u, v)=\frac{\mathbf{F}^{t}(u) \mathbf{H}(v)}{u-v} .
$$

It turns out that this resolvent kernel is related to the following $\mathrm{RH}$ problem.

RH problem 3.1. Let $s>0$ and $\lambda>0$. We look for a $2 \times 2$ matrix-valued function $Y(z)$ satisfying

(1) $Y(z)$ is defined and analytic in $\mathbb{C} \backslash[-s, 0]$, where the orientation is taken from the left to the right.

(2) For $x \in(-s, 0)$, we have

$$
Y_{+}(x)=Y_{-}(x)\left(I-2 \pi i \mathbf{f}(x) \mathbf{h}^{t}(x)\right),
$$

where $\mathbf{f}$ and $\mathbf{h}$ also depend on the parameter $\lambda$ by (3.4).

(3) As $z \rightarrow \infty$,

$$
Y(z)=I+O(1 / z)
$$

(4) We have

$$
Y(z)= \begin{cases}O(1), & \text { as } z \rightarrow 0, \\ O(\ln (z+s)), & \text { as } z \rightarrow-s\end{cases}
$$

By [19, it follows that

$$
Y(z)=I-\int_{-s}^{0} \frac{\mathbf{F}(w) \mathbf{h}^{t}(w)}{w-z} d w
$$

and

$$
\mathbf{F}(z)=Y(z) \mathbf{f}(z), \quad \mathbf{H}(z)=\left(Y^{t}(z)\right)^{-1} \mathbf{h}(z) .
$$


Recalling the $\mathrm{RH}$ problem 1.1 for $\Psi$, we make the following undressing transformation to arrive at a $\mathrm{RH}$ problem with constant jumps. Define

$$
X(z ; \lambda, s)=X(z)=\left\{\begin{array}{cl}
Y(z) \Psi(z), & \text { for } z \text { in region } \mathrm{I} \cup \mathrm{III} \cup \mathrm{IV}, \\
Y(z) \Psi(z)\left(\begin{array}{cc}
1 & 0 \\
e^{\alpha \pi i} & 1
\end{array}\right), & \text { for } z \text { in region II, } \\
Y(z) \Psi(z)\left(\begin{array}{rr}
1 & 0 \\
-e^{-\alpha \pi i} & 1
\end{array}\right), & \text { for } z \text { in region } \mathrm{V},
\end{array}\right.
$$

where the regions $\mathrm{I}-\mathrm{V}$ are shown in Figure 2, Then, $X$ satisfies the following $\mathrm{RH}$ problem.

Proposition 3.2. The function $X$ defined in (3.13) has the following properties:

(1) $X(z)$ is defined and analytic in $\mathbb{C} \backslash\left\{\cup_{j=1}^{4} \Gamma_{j}^{(s)} \cup\{-s\}\right\}$, where

$$
\Gamma_{1}^{(s)}=(-s, 0), \quad \Gamma_{2}^{(s)}=-s+e^{-\pi i / 3} \mathbb{R}^{-}, \quad \Gamma_{3}^{(s)}=(-\infty,-s), \quad \Gamma_{4}^{(s)}=-s+e^{\pi i / 3} \mathbb{R}^{-},
$$

with $\mathbb{R}^{-}:=(-\infty, 0)$ and all orientations from the left to the right; see the solid lines in Figure Q

(2) X satisfies the following jump conditions:

$$
X_{+}(z)=X_{-}(z) \begin{cases}\left(\begin{array}{cc}
e^{\alpha \pi i} & 0 \\
0 & e^{-\alpha \pi i}
\end{array}\right), & z \in \Gamma_{1}^{(s)}, \\
\left(\begin{array}{cc}
1 & 0 \\
e^{\alpha \pi i} & 1
\end{array}\right), & z \in \Gamma_{2}^{(s)}, \\
\left(\begin{array}{cc}
0 & 1 \\
-1 & 0
\end{array}\right), & z \in \Gamma_{3}^{(s)}, \\
\left(\begin{array}{cc}
1 & 0 \\
e^{-\alpha \pi i} & 1
\end{array}\right), & z \in \Gamma_{4}^{(s)} .\end{cases}
$$

(3) As $z \rightarrow \infty$,

$$
X(z)=\left(I+\frac{X_{\infty}}{z}+O\left(z^{-2}\right)\right) z^{-\frac{1}{4} \sigma_{3}} \frac{I+i \sigma_{1}}{\sqrt{2}} e^{z^{\frac{1}{2}} \sigma_{3}} .
$$

(4) We have

$$
X(z)= \begin{cases}O(1)(I+O(z)) e^{(-1)^{k+1}\left(\frac{\lambda}{z}\right)^{k} \sigma_{3}} z^{\frac{\alpha}{2} \sigma_{3}}, & \text { as } z \rightarrow 0, \\ O(\ln (z+s)), & \text { as } z \rightarrow-s .\end{cases}
$$

Proof. It suffices to check the jump condition on $\Gamma_{1}^{(s)}$, while the other claims follow directly from the definition (3.13).

By (1.13) and (3.4), we have, for $x<0$,

$$
\mathbf{f}(x)=\Psi_{-}(x)\left(\begin{array}{c}
-e^{\frac{\alpha \pi i}{2}} \\
e^{-\frac{\alpha \pi i}{2}}
\end{array}\right), \quad \mathbf{h}(x)=-\frac{1}{2 \pi i} \Psi_{-}^{-t}(x)\left(\begin{array}{c}
e^{-\frac{\alpha \pi i}{2}} \\
e^{\frac{\alpha \pi i}{2}}
\end{array}\right) .
$$


Thus,

$$
I-2 \pi i \mathbf{f}(x) \mathbf{h}^{t}(x)=\Psi_{-}(x)\left(\begin{array}{cc}
0 & -e^{\alpha \pi i} \\
e^{-\alpha \pi i} & 2
\end{array}\right) \Psi_{-}^{-1}(x) .
$$

This, together with (3.8) and (3.13), implies that if $-s<x<0$,

$$
\begin{aligned}
X_{+}(x) & =Y_{+}(x) \Psi_{+}(x)\left(\begin{array}{cc}
1 & 0 \\
e^{\alpha \pi i} & 1
\end{array}\right) \\
& =Y_{-}(x) \Psi_{-}(x)\left(\begin{array}{cc}
0 & -e^{\alpha \pi i} \\
e^{-\alpha \pi i} & 2
\end{array}\right) \Psi_{-}^{-1}(x) \Psi_{+}(x)\left(\begin{array}{cc}
1 & 0 \\
e^{\alpha \pi i} & 1
\end{array}\right) \\
& =X_{-}(x)\left(\begin{array}{cc}
1 & 0 \\
e^{-\alpha \pi i} & 1
\end{array}\right)\left(\begin{array}{cc}
0 & -e^{\alpha \pi i} \\
e^{-\alpha \pi i} & 2
\end{array}\right)\left(\begin{array}{cc}
0 & 1 \\
-1 & 0
\end{array}\right)\left(\begin{array}{cc}
1 & 0 \\
e^{\alpha \pi i} & 1
\end{array}\right) \\
& =X_{-}(x)\left(\begin{array}{cc}
e^{\alpha \pi i} & 0 \\
0 & e^{-\alpha \pi i}
\end{array}\right),
\end{aligned}
$$

as desired.

Remark 3.3. Solvability of the RH problem for $X$ follows from (6.1) and Theorem 6.3 below.

The connection between $X$ and $\frac{\partial}{\partial s} F(s ; \lambda)$ is stated in the following proposition.

Proposition 3.4. We have

$$
\frac{\partial}{\partial s} F(s ; \lambda)=\frac{d}{d s} \ln \operatorname{det}\left(I-\widetilde{\mathcal{K}}_{P I I I}\right)=-\frac{e^{\alpha \pi i}}{2 \pi i} \lim _{z \rightarrow-s}\left(X^{-1}(z) X^{\prime}(z)\right)_{21},
$$

where the limit is taken as $z$ tends to $-s$ in region $V$.

Proof. For $z$ belonging to region V, we see from (3.12), (3.13) and (3.18) that

$$
\mathbf{F}(z)=X(z)\left(\begin{array}{c}
-e^{\alpha \pi i / 2} \\
0
\end{array}\right), \quad \mathbf{H}(z)=-\frac{X^{-t}(z)}{2 \pi i}\left(\begin{array}{c}
0 \\
e^{\alpha \pi i / 2}
\end{array}\right) .
$$

A combination of L'Hôpital's rule, (3.1), (3.5), (3.7) and (3.22) then gives us (3.21).

\subsection{Asymptotic analysis of the Riemann-Hilbert problem for $X$}

In this section, we shall perform the Deift-Zhou steepest descent analysis to the RH problem for $X$ as $s \rightarrow+\infty$. It consists of a series of explicit and invertible transformations which leads to a $\mathrm{RH}$ problem tending to the identity matrix as $s \rightarrow+\infty$.

\subsection{1 $X \rightarrow T$ : Rescaling}

Define

$$
T(z)=X(s z) .
$$

It is then straightforward to check that $T$ satisfies a $\mathrm{RH}$ problem as follows: 


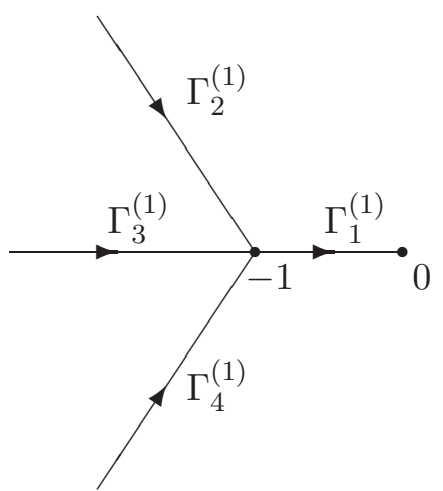

Figure 3: Contours $\Gamma_{j}^{(1)}, j=1,2,3,4$, for the $\mathrm{RH}$ problem for $T$.

RH problem 3.5. The function $T$ defined in (3.23) has the following properties:

(1) $T(z)$ is defined and analytic in $\mathbb{C} \backslash\left\{\cup_{j=1}^{4} \Gamma_{j}^{(1)} \cup\{-1\}\right\}$, where the contours $\Gamma_{j}^{(1)}$ are defined in (3.14) with $s=1$, as shown in Figure 3 .

(2) T satisfies the following jump conditions:

$$
T_{+}(z)=T_{-}(z)\left\{\begin{array}{l}
\left(\begin{array}{cc}
e^{\alpha \pi i} & 0 \\
0 & e^{-\alpha \pi i}
\end{array}\right), \quad z \in \Gamma_{1}^{(1)}, \\
\left(\begin{array}{cc}
1 & 0 \\
e^{\alpha \pi i} & 1
\end{array}\right), \\
\left(\begin{array}{cc}
0 & 1 \\
-1 & 0
\end{array}\right), \\
\left(\begin{array}{cc}
1 & 0 \\
e^{-\alpha \pi i} & 1
\end{array}\right), \quad z \in \Gamma_{2}^{(1)},
\end{array}\right.
$$

(3) As $z \rightarrow \infty$,

$$
T(z)=\left(I+O\left(\frac{1}{z}\right)\right)(s z)^{-\frac{1}{4} \sigma_{3}} \frac{I+i \sigma_{1}}{\sqrt{2}} e^{(s z)^{\frac{1}{2}} \sigma_{3}} .
$$

(4) For $z \notin(-\infty, 0]$, we have

$$
T(z)= \begin{cases}O(1)(I+O(z)) e^{(-1)^{k+1}\left(\frac{\lambda}{s z}\right)^{k} \sigma_{3} z^{\frac{\alpha}{2} \sigma_{3}},}, & \text { as } z \rightarrow 0, \\ O(\ln (z+1)), & \text { as } z \rightarrow-1 .\end{cases}
$$

3.2.2 $T \rightarrow S$ : Normalization at $\infty$ and 0

Define

$$
g(z)=\sqrt{z+1}\left(1+(-1)^{k+1} \frac{\lambda^{k}}{s^{k+1 / 2}} \sum_{j=1}^{k} \frac{c_{j}}{z^{j}}\right), \quad z \in \mathbb{C} \backslash(-\infty,-1],
$$


where

$$
c_{k}=1 \quad \text { and } \quad \sum_{j=0}^{m}\left(\begin{array}{c}
1 / 2 \\
m-j
\end{array}\right) c_{k-j}=0 \quad \text { for } m=1, \ldots, k-1 .
$$

From the above definition, it is readily seen that

$$
g_{+}(x)+g_{-}(x)=0 \quad \text { for } x<-1,
$$

and

$$
g(z)= \begin{cases}\sqrt{z}+\mathfrak{g}_{1} z^{-1 / 2}+O\left(z^{-3 / 2}\right), & \text { as } z \rightarrow \infty \\ (-1)^{k+1} \frac{\lambda^{k}}{s^{k+1 / 2} z^{k}}+O(1), & \text { as } z \rightarrow 0\end{cases}
$$

with

$$
\mathfrak{g}_{1}=\frac{1}{2}+(-1)^{k+1} \frac{\lambda^{k}}{s^{k+1 / 2}} c_{1} .
$$

The second transformation is then defined by

$$
S(z)=s^{\frac{1}{4} \sigma_{3}}\left(\begin{array}{cc}
1 & 0 \\
i s \mathfrak{g}_{1} & 1
\end{array}\right) T(z) e^{-\sqrt{s} g(z) \sigma_{3}},
$$

where $\mathfrak{g}_{1}$ is given in (3.30). It is straightforward to check that $S$ satisfies the following RH problem.

RH problem 3.6. The function $S$ defined in (3.31) has the following properties:

(1) $S(z)$ is defined and analytic in $\mathbb{C} \backslash\left\{\cup_{j=1}^{4} \Gamma_{j}^{(1)} \cup\{-1\}\right\}$; see Figure .

(2) S satisfies the following jump conditions:

$$
S_{+}(z)=S_{-}(z) \begin{cases}\left(\begin{array}{cc}
e^{\alpha \pi i} & 0 \\
0 & e^{-\alpha \pi i}
\end{array}\right), & z \in \Gamma_{1}^{(1)}, \\
\left(\begin{array}{cc}
e^{\alpha \pi i-2 \sqrt{s} g(z)} & 1
\end{array}\right), & z \in \Gamma_{2}^{(1)}, \\
\left(\begin{array}{cc}
0 & 1 \\
-1 & 0
\end{array}\right), & \\
\left(\begin{array}{cc}
1 & 0 \\
e^{-\alpha \pi i-2 \sqrt{s} g(z)} & 1
\end{array}\right), & z \in \Gamma_{3}^{(1)},\end{cases}
$$

(3) $A s z \rightarrow \infty$,

$$
S(z)=\left(I+O\left(\frac{1}{z}\right)\right) z^{-\frac{1}{4} \sigma_{3}} \frac{I+i \sigma_{1}}{\sqrt{2}} .
$$

(4) We have

$$
S(z)= \begin{cases}O(1) z^{\frac{\alpha}{2} \sigma_{3}}, & \text { as } z \rightarrow 0 \\ O(\ln (z+1)), & \text { as } z \rightarrow-1\end{cases}
$$


By (3.27), one verifies that for sufficiently large positive $s$,

$$
\operatorname{Re} g(z)>0, \quad z \in \Gamma_{2}^{(1)} \cup \Gamma_{4}^{(1)} .
$$

Hence, the jump matrix of $S$ on $\Gamma_{2}^{(1)} \cup \Gamma_{4}^{(1)}$ tends to the identity matrix exponentially fast as $s \rightarrow+\infty$. This uniform convergence is not valid anymore for $z$ near -1 .

\subsubsection{Outer parametrix}

By ignoring the exponentially small terms in the jumps (3.32) and a neighborhood of -1 , we are led to the following $\mathrm{RH}$ problem for the outer parametrix $N$.

RH problem 3.7. We look for a $2 \times 2$ matrix-valued function $N(z)$ satisfying

(1) $N(z)$ is defined and analytic in $\mathbb{C} \backslash(-\infty, 0]$.

(2) For $x<0$, we have

$$
N_{+}(x)=N_{-}(x) \begin{cases}\left(\begin{array}{cc}
e^{\alpha \pi i} & 0 \\
0 & e^{-\alpha \pi i}
\end{array}\right), & x \in(-1,0), \\
\left(\begin{array}{cc}
0 & 1 \\
-1 & 0
\end{array}\right), & x \in(-\infty,-1) .\end{cases}
$$

(3) As $z \rightarrow \infty$,

$$
N(z)=\left(I+O\left(\frac{1}{z}\right)\right) z^{-\frac{1}{4} \sigma_{3}} \frac{I+i \sigma_{1}}{\sqrt{2}}
$$

(4) As $z \rightarrow 0$,

$$
N(z)=O(1) z^{\frac{\alpha}{2} \sigma_{3}}
$$

The above $\mathrm{RH}$ problem can be solved explicitly and the solution is given by (cf. [28])

$$
N(z)=\left(\begin{array}{cc}
1 & 0 \\
i \alpha & 1
\end{array}\right)(z+1)^{-\frac{1}{4} \sigma_{3}} \frac{1}{\sqrt{2}}\left(I+i \sigma_{1}\right)\left(\frac{\sqrt{z+1}+1}{\sqrt{z+1}-1}\right)^{-\frac{\alpha}{2} \sigma_{3}}, z \in \mathbb{C} \backslash(-\infty, 0],
$$

where the branches are chosen $\operatorname{as} \arg (z+1) \in(-\pi, \pi), \arg z \in(-\pi, \pi)$, and such that the last factor in (3.38) behaves

$$
\left(\frac{\sqrt{z+1}+1}{\sqrt{z+1}-1}\right)^{-\frac{\alpha}{2} \sigma_{3}}=\left(1+\frac{\alpha^{2}}{2 z}\right) I-\frac{\alpha}{\sqrt{z}} \sigma_{3}+O\left(z^{-3 / 2}\right) \quad \text { as } z \rightarrow \infty .
$$

\subsubsection{Local parametrix near -1}

Let $D_{-1}$ be a small, open neighborhood near -1 with fixed radius. Due to the fact that the convergence of the jump matrices to the identity matrices on $\Gamma_{2}^{(1)} \cup \Gamma_{4}^{(1)}$ is not uniform near -1 , we intend to find a function $P_{-1}$ satisfying a $\mathrm{RH}$ problem as follows.

RH problem 3.8. We look for a $2 \times 2$ matrix-valued function $P_{-1}(z)$ satisfying

(1) $P_{-1}(z)$ is defined and analytic in $\overline{D_{-1}} \backslash\left\{\cup_{j=1}^{4} \Gamma_{j}^{(1)} \cup\{-1\}\right\}$. 
(2) $P_{-1}(z)$ satisfies the same jump conditions (3.32) as $S$ for $z \in D_{-1} \cap\left\{\cup_{j=1}^{4} \Gamma_{j}^{(1)}\right\}$.

(3) As $s \rightarrow+\infty, P_{-1}$ matches $N(z)$ on the boundary $\partial D_{-1}$ of $D_{-1}$, i.e.,

$$
P_{-1}(z)=N(z)(I+o(1)), \quad z \in \partial D_{-1} .
$$

To solve the above $\mathrm{RH}$ problem, we note that, as $z \rightarrow-1$,

$$
g(z)=\sqrt{z+1}\left[1-\frac{\lambda^{k}}{s^{k+1 / 2}}\left(\eta_{0}+\eta_{1}(z+1)+O(z+1)^{2}\right)\right]
$$

with

$$
\eta_{0}=\sum_{j=1}^{k}(-1)^{k+j} c_{j}, \quad \eta_{1}=\sum_{j=1}^{k}(-1)^{k+j} j c_{j},
$$

where $c_{j}$ are determined in (3.28). This local behavior suggests us to construct $P_{-1}$ with the help of the Bessel parametrix $\Phi_{\text {Bes }}^{(\alpha)}$, which solves the following model RH problem.

RH problem 3.9. The $2 \times 2$ matrix-valued function $\Phi_{\text {Bes }}^{(\alpha)}(\zeta)$ has the following properties:

(1) $\Phi_{\text {Bes }}^{(\alpha)}(\zeta)$ is defined and analytic in $\mathbb{C} \backslash\left\{\cup_{j=1}^{3} \Sigma_{j} \cup\{0\}\right\}$, where the contours $\Sigma_{j}, j=1,2,3$, are illustrated in Figure 1 .

(2) $\Phi_{\text {Bes }}^{(\alpha)}(\zeta)$ satisfies the following jump conditions:

$$
\Phi_{\text {Bes, },+}^{(\alpha)}(\zeta)=\Phi_{\text {Bes, }-}^{(\alpha)}(\zeta)\left\{\begin{array}{l}
\left(\begin{array}{cc}
1 & 0 \\
e^{\alpha \pi i} & 1
\end{array}\right), \quad \zeta \in \Sigma_{1}, \\
\left(\begin{array}{cc}
0 & 1 \\
-1 & 0
\end{array}\right), \quad \zeta \in \Sigma_{2}, \\
\left(\begin{array}{cc}
1 & 0 \\
e^{-\alpha \pi i} & 1
\end{array}\right), \quad \zeta \in \Sigma_{3} .
\end{array}\right.
$$

(3) $A s \zeta \rightarrow \infty$,

$$
\Phi_{\text {Bes }}^{(\alpha)}(\zeta)=\left(4 \pi^{2} \zeta\right)^{-\frac{1}{4} \sigma_{3}} \frac{I+i \sigma_{1}}{\sqrt{2}}\left(I+\frac{1}{16 \sqrt{\zeta}}\left(\begin{array}{cc}
-1-4 \alpha^{2} & -2 i \\
-2 i & 1+4 \alpha^{2}
\end{array}\right)+O\left(\frac{1}{\zeta}\right)\right) e^{2 \sqrt{\zeta} \sigma_{3}}
$$

(4) The matrix function $\Phi_{\text {Bes }}^{(\alpha)}(\zeta)$ has the following local behavior near the origin. If $\alpha<0$,

$$
\Phi_{\text {Bes }}^{(\alpha)}(\zeta)=O\left(\begin{array}{ll}
|\zeta|^{\alpha / 2} & |\zeta|^{\alpha / 2} \\
|\zeta|^{\alpha / 2} & |\zeta|^{\alpha / 2}
\end{array}\right), \quad \text { as } \zeta \rightarrow 0
$$

If $\alpha=0$,

$$
\Phi_{\text {Bes }}^{(\alpha)}(\zeta)=O\left(\begin{array}{ll}
\ln |\zeta| & \ln |\zeta| \\
\ln |\zeta| & \ln |\zeta|
\end{array}\right), \quad \text { as } \zeta \rightarrow 0
$$


If $\alpha>0$,

$$
\Phi_{\text {Bes }}^{(\alpha)}(\zeta)=\left\{\begin{array}{l}
O\left(\begin{array}{ll}
|\zeta|^{\alpha / 2} & |\zeta|^{-\alpha / 2} \\
|\zeta|^{\alpha / 2} & |\zeta|^{-\alpha / 2}
\end{array}\right), \quad \text { as } \zeta \rightarrow 0 \text { and }|\arg \zeta|<2 \pi / 3, \\
O\left(\begin{array}{ll}
|\zeta|^{-\alpha / 2} & |\zeta|^{-\alpha / 2} \\
|\zeta|^{-\alpha / 2} & |\zeta|^{-\alpha / 2}
\end{array}\right), \quad \text { as } \zeta \rightarrow 0 \text { and } 2 \pi / 3<|\arg \zeta|<\pi .
\end{array}\right.
$$

By [31], the solution to $\mathrm{RH}$ problem 3.9 is given by

$$
\Phi_{\mathrm{Bes}}^{(\alpha)}(\zeta)=\left\{\begin{array}{cc}
\left(\begin{array}{cc}
I_{\alpha}\left(2 \zeta^{1 / 2}\right) & \frac{i}{\pi} K_{\alpha}\left(2 \zeta^{1 / 2}\right) \\
2 \pi i \zeta^{1 / 2} I_{\alpha}^{\prime}\left(2 \zeta^{1 / 2}\right) & -2 \zeta^{1 / 2} K_{\alpha}^{\prime}\left(2 \zeta^{1 / 2}\right)
\end{array}\right), & \text { for }|\arg \zeta|<2 \pi / 3, \\
\left(\begin{array}{cc}
I_{\alpha}\left(2 \zeta^{1 / 2}\right) & \frac{i}{\pi} K_{\alpha}\left(2 \zeta^{1 / 2}\right) \\
2 \pi i \zeta^{1 / 2} I_{\alpha}^{\prime}\left(2 \zeta^{1 / 2}\right) & -2 \zeta^{1 / 2} K_{\alpha}^{\prime}\left(2 \zeta^{1 / 2}\right)
\end{array}\right)\left(\begin{array}{cc}
1 & 0 \\
-e^{\alpha \pi i} & 1
\end{array}\right), & \text { for } 2 \pi / 3<\arg \zeta<\pi, \\
\left(\begin{array}{cc}
I_{\alpha}\left(2 \zeta^{1 / 2}\right) & \frac{i}{\pi} K_{\alpha}\left(2 \zeta^{1 / 2}\right) \\
2 \pi i \zeta^{1 / 2} I_{\alpha}^{\prime}\left(2 \zeta^{1 / 2}\right) & -2 \zeta^{1 / 2} K_{\alpha}^{\prime}\left(2 \zeta^{1 / 2}\right)
\end{array}\right)\left(\begin{array}{cc}
1 & 0 \\
e^{-\alpha \pi i} & 1
\end{array}\right), & \text { for }-\pi<\arg \zeta<-2 \pi / 3 .
\end{array}\right.
$$

where $I_{\alpha}$ and $K_{\alpha}$ denote the usual modified Bessel functions [1] with a cut along the negative real axis.

Recalling the local behaviour of $S(z)$ near -1 in (3.34), the local parametrix $P_{-1}(z)$ is constructed in terms of the Bessel parametrix of order 0 as follows:

$$
P_{-1}(z)=E_{-1}(z) \Phi_{\text {Bes }}^{(0)}\left(\frac{1}{4} s g^{2}(z)\right) \begin{cases}e^{\left(-\sqrt{s} g(z)+\frac{\alpha \pi i}{2}\right) \sigma_{3}}, & \text { for } D_{-1} \cap\{z \mid \operatorname{Im} z>0\} \\ e^{\left(-\sqrt{s} g(z)-\frac{\alpha \pi i}{2}\right) \sigma_{3}}, & \text { for } D_{-1} \cap\{z \mid \operatorname{Im} z<0\}\end{cases}
$$

where

$$
E_{-1}(z):=N(z) \begin{cases}e^{-\frac{\alpha \pi i}{2} \sigma_{3}} \frac{I-i \sigma_{1}}{\sqrt{2}}\left(\pi^{2} s g^{2}(z)\right)^{\frac{1}{4} \sigma_{3}}, & \text { for } \operatorname{Im} z>0 \\ e^{\frac{\alpha \pi i}{2} \sigma_{3}} \frac{I-i \sigma_{1}}{\sqrt{2}}\left(\pi^{2} s g^{2}(z)\right)^{\frac{1}{4} \sigma_{3}}, & \text { for } \operatorname{Im} z<0\end{cases}
$$

and $N(z)$ is defined in (3.38).

To show $P_{-1}(z)$ defined in (3.49) indeed satisfies $\mathrm{RH}$ problem [3.8, we first note from (3.41) that $g^{2}(z)$ is a conformal mapping of $D_{-1}$ that maps -1 to 0 , preserves the real line, and maps $\Gamma_{2}^{(1)} \cup \Gamma_{4}^{(1)}$ onto $\Sigma_{1} \cup \Sigma_{3}$. It is then straightforward to check from (3.49) and (3.43) that $P_{-1}$ satisfies the jump conditions stated in item (2) of RH problem 3.8, provided we can show the prefactor $E_{-1}(z)$ is analytic in $D_{-1}$. To see this, it suffices to check that the prefactor has no jump on $D_{-1} \cap\left\{\Gamma_{1}^{(1)} \cup \Gamma_{3}^{(1)}\right\}$. Note that $g^{1 / 2}(z)$ is analytic in $\mathbb{C} \backslash(-\infty,-1]$, if $x \in D_{-1} \cap \Gamma_{1}^{(1)}$, we see from (3.50) and (3.35) that

$$
\begin{aligned}
E_{-1,+}(x) & =N_{+}(x) e^{-\frac{\alpha \pi i}{2} \sigma_{3}} \frac{I-i \sigma_{1}}{\sqrt{2}}\left(\pi^{2} s g^{2}(x)\right)^{\frac{1}{4} \sigma_{3}} \\
& =N_{-}(x) e^{+\frac{\alpha \pi i}{2} \sigma_{3}} \frac{I-i \sigma_{1}}{\sqrt{2}}\left(\pi^{2} s g^{2}(x)\right)^{\frac{1}{4} \sigma_{3}}=E_{-1,-}(x) .
\end{aligned}
$$


If $x \in D_{-1} \cap \Gamma_{3}^{(1)}$, again by (3.50) and (3.35), it follows that

$$
\begin{aligned}
& E_{-1,+}(x) E_{-1,-}^{-1}(x) \\
& =N_{+}(x) e^{-\frac{\alpha \pi i}{2} \sigma_{3}} \frac{I-i \sigma_{1}}{\sqrt{2}}\left(\pi^{2} s g^{2}(x)\right)^{\frac{1}{4} \sigma_{3}}\left(\pi^{2} s g^{2}(x)\right)^{-\frac{1}{4} \sigma_{3}} \frac{I+i \sigma_{1}}{\sqrt{2}} e^{-\frac{\alpha \pi i}{2} \sigma_{3}} N_{-}^{-1}(x) \\
& =N_{+}(x) e^{-\frac{\alpha \pi i}{2} \sigma_{3}} \frac{I-i \sigma_{1}}{\sqrt{2}}\left(\begin{array}{cc}
i & 0 \\
0 & -i
\end{array}\right) \frac{I+i \sigma_{1}}{\sqrt{2}} e^{-\frac{\alpha \pi i}{2} \sigma_{3}}\left(\begin{array}{cc}
0 & 1 \\
-1 & 0
\end{array}\right) N_{+}^{-1}(x) \\
& =N_{+}(x) e^{-\frac{\alpha \pi i}{2} \sigma_{3}}\left(\begin{array}{cc}
0 & -1 \\
1 & 0
\end{array}\right) e^{-\frac{\alpha \pi i}{2} \sigma_{3}}\left(\begin{array}{cc}
0 & 1 \\
-1 & 0
\end{array}\right) N_{+}^{-1}(x)=I .
\end{aligned}
$$

Finally, for the matching condition (3.40), we observe that

$$
\left(\frac{\sqrt{z+1}+1}{\sqrt{z+1}-1}\right)^{-\frac{\alpha}{2}}=\rho(z) \begin{cases}e^{\frac{\alpha \pi i}{2}}, & \operatorname{Im} z>0 \\ e^{-\frac{\alpha \pi i}{2}}, & \operatorname{Im} z<0\end{cases}
$$

where

$$
\rho(z):=\left(\frac{1-\sqrt{z+1}}{1+\sqrt{z+1}}\right)^{\alpha / 2}, \quad z \in \mathbb{C} \backslash((-\infty,-1],[0,+\infty)) .
$$

This, together with (3.38), (3.44) and (3.49) and a straightforward calculation, implies that, for $z \in \partial D_{-1}$ and large positive $s$,

$$
\begin{aligned}
& P_{-1}(z) N(z)^{-1} \\
& =N(z) e^{\mp \frac{\alpha \pi i}{2} \sigma_{3}} \frac{I-i \sigma_{1}}{\sqrt{2}}\left(\pi^{2} s g^{2}(z)\right)^{\frac{1}{4} \sigma_{3}} \Phi_{\mathrm{Bes}}^{(0)}\left(\frac{1}{4} s g^{2}(z)\right) e^{-\sqrt{s} g(z) \sigma_{3}} e^{ \pm \frac{\alpha \pi i}{2} \sigma_{3}} N(z)^{-1} \\
& =I+\frac{1}{s^{1 / 2}}\left(\begin{array}{cc}
1 & 0 \\
i \alpha & 1
\end{array}\right) J_{1}(z)\left(\begin{array}{cc}
1 & 0 \\
-i \alpha & 1
\end{array}\right)+O\left(s^{-1}\right),
\end{aligned}
$$

where

$$
J_{1}(z)=\left(\begin{array}{cc}
\frac{\rho(z)^{-2}-\rho(z)^{2}}{8 g(z)} & \frac{-i\left(\left(\rho(z)+\rho(z)^{-1}\right)^{2}-3\right)}{8 \sqrt{z+1} g(z)} \\
\frac{-i\left(\left(\rho(z)+\rho(z)^{-1}\right)^{2}-1\right) \sqrt{z+1}}{8 g(z)} & \frac{\rho(z)^{2}-\rho(z)^{-2}}{8 g(z)}
\end{array}\right),
$$

as required.

We conclude this section by evaluating $E_{-1}(-1)$ for later use. By (3.38) and (3.50), it is readily seen that

$$
E_{-1}(z)=\left(\begin{array}{cc}
1 & 0 \\
i \alpha & 1
\end{array}\right) \widetilde{E}_{-1}(z)
$$

where

$$
\begin{aligned}
\widetilde{E}_{-1}(z) & =(z+1)^{-\frac{1}{4} \sigma_{3}} \frac{I+i \sigma_{1}}{\sqrt{2}} \rho(z)^{\sigma_{3}} \frac{I-i \sigma_{1}}{\sqrt{2}}\left(\pi^{2} s g^{2}(z)\right)^{\frac{1}{4} \sigma_{3}} \\
& =(z+1)^{-\frac{1}{4} \sigma_{3}}\left(\frac{\rho(z)+\rho^{-1}(z)}{2} I+\frac{\rho(z)-\rho^{-1}(z)}{2} \sigma_{2}\right)\left(\pi^{2} s g^{2}(z)\right)^{\frac{1}{4} \sigma_{3}}
\end{aligned}
$$




$$
\begin{aligned}
& \text { with } \sigma_{2}=\left(\begin{array}{cc}
0 & -i \\
i & 0
\end{array}\right) \text {. Since } \\
& \qquad \begin{array}{l}
\rho(z)=1-\alpha(z+1)^{\frac{1}{2}}+\frac{\alpha^{2}}{2}(z+1)+O\left((z+1)^{3 / 2}\right), \quad z \rightarrow-1,
\end{array}
\end{aligned}
$$

it then follows from (3.59) and the above formula that

$$
\widetilde{E}_{-1}(-1)=\left(\begin{array}{cc}
\sqrt{\pi}\left(\frac{s^{k+1 / 2}-\lambda^{k} \eta_{0}}{s^{k}}\right)^{1 / 2} & \frac{i \alpha}{\sqrt{\pi}}\left(\frac{s^{k}}{s^{k+1 / 2}-\lambda^{k} \eta_{0}}\right)^{1 / 2} \\
0 & \frac{1}{\sqrt{\pi}}\left(\frac{s^{k}}{s^{k+1 / 2}-\lambda^{k} \eta_{0}}\right)^{1 / 2}
\end{array}\right),
$$

and

$$
\widetilde{E}_{-1}^{\prime}(-1)=\left(\begin{array}{cc}
* & * \\
-i \alpha \sqrt{\pi}\left(\frac{s^{k+1 / 2}-\lambda^{k} \eta_{0}}{s^{k}}\right)^{1 / 2} & *
\end{array}\right),
$$

where $\eta_{0}$ is given in (3.42) and $*$ denotes certain unimportant entries.

\subsubsection{Final transformation}

Our final transformation is defined by

$$
R(z)= \begin{cases}S(z) N(z)^{-1}, & \text { for } z \in \mathbb{C} \backslash D_{-1}, \\ S(z) P_{-1}(z)^{-1}, & \text { for } z \in D_{-1} .\end{cases}
$$

It is then easily seen that $R$ satisfies the following $\mathrm{RH}$ problem.

RH problem 3.10. The $2 \times 2$ matrix-valued function $R(z)$ defined in (3.63) has the following properties:

(1) $R(z)$ is analytic in $\mathbb{C} \backslash \Sigma_{R}$, where the contour $\Sigma_{R}$ is shown in Figure 4 .

(2) $R(z)$ satisfies the jump condition

$$
R_{+}(z)=R_{-}(z) J_{R}(z), \quad z \in \Sigma_{R},
$$

where

$$
J_{R}(z)= \begin{cases}P_{-1}(z) N(z)^{-1}, & \text { for } z \in \partial D_{-1}, \\ N(z) J_{S}(z) N(z)^{-1}, & \text { for } z \in \Sigma_{R} \backslash \partial D_{-1} .\end{cases}
$$

(3) As $z \rightarrow \infty$,

$$
R(z)=I+O(1 / z) .
$$

For $z \in \Sigma_{R} \backslash \overline{D_{-1}}$, there exits some constant $c>0$ such that

$$
J_{R}(z)=I+O\left(e^{-c \sqrt{s}}\right), \quad \text { as } s \rightarrow+\infty,
$$

uniformly valid for $\lambda$ in any compact subset of $(0,+\infty)$. This, together with (3.56) and standard analysis (cf. [18, 22]), implies that $R(z)$ admits an asymptotic expansion of the following form

$$
R(z)=I+\frac{1}{s^{1 / 2}}\left(\begin{array}{cc}
1 & 0 \\
\alpha i & 1
\end{array}\right) R_{1}(z)\left(\begin{array}{cc}
1 & 0 \\
-\alpha i & 1
\end{array}\right)+O\left(s^{-1}\right)
$$




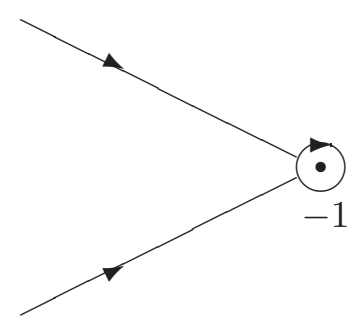

Figure 4: Contour $\Sigma_{R}$ for the RH problem 3.10 for $R$.

uniformly for $z \in \mathbb{C} \backslash \Sigma_{R}$ on compact subsets.

Next, a combination of (3.66) and RH problem 3.10 shows that $R_{1}(z)$ is a solution of the following $\mathrm{RH}$ problem.

RH problem 3.11. The $2 \times 2$ matrix-valued function $R_{1}(z)$ appearing in (3.66) has the following properties:

(1) $R_{1}(z)$ is analytic in $\mathbb{C} \backslash \partial D_{-1}$.

(2) For $z \in \partial D_{-1}$, we have

$$
R_{1,+}(z)-R_{1,-}(z)=J_{1}(z),
$$

where $J_{1}(z)$ is given in (3.57).

(3) As $z \rightarrow \infty, R_{1}(z)=O(1 / z)$.

By Cauchy theorem and the residue theorem, we obtain that

$$
R_{1}(z)=\frac{1}{2 \pi i} \oint_{\partial D_{-1}} \frac{J_{1}(\zeta)}{\zeta-z} d \zeta=\left\{\begin{array}{cc}
\left(\begin{array}{cc}
0 & \frac{1}{8 i(z+1)\left(1-\frac{\lambda^{k} \eta_{0}}{s^{k+1 / 2}}\right)} \\
0 & 0 \\
0 & \frac{1}{8 i(z+1)\left(1-\frac{\lambda^{k} \eta_{0}}{s^{k+1 / 2}}\right)} \\
0 & 0
\end{array}\right), \quad \text { for } z \in \mathbb{C} \backslash D_{-1} .
\end{array}\right.
$$

Furthermore, we note that

$$
\begin{aligned}
\left(R_{1}(z)\right)_{21}= & \frac{i\left(\left(\rho(z)+\rho(z)^{-1}\right)^{2}-1\right) \sqrt{z+1}}{8 g(z)} \\
= & \frac{3 i s^{k+1 / 2}}{8\left(s^{k+1 / 2}-\lambda^{k} \eta_{0}\right)}+i\left[\frac{\alpha^{2}}{2} \frac{s^{k+1 / 2}}{s^{k+1 / 2}-\lambda^{k} \eta_{0}}+\frac{3 \eta_{1}}{8} \frac{\lambda^{k} s^{k+1 / 2}}{\left(s^{k+1 / 2}-\lambda^{k} \eta_{0}\right)^{2}}\right](z+1) \\
& +O(z+1)^{2}
\end{aligned}
$$

as $z \rightarrow-1$, where the constants $\eta_{0}$ and $\eta_{1}$ are given in (3.42). 


\subsection{Large $s$ asymptotics of $\frac{\partial}{\partial s} F(s ; \lambda)$}

Tracing back the transformations $S \mapsto T \mapsto X$, we have from (3.21), (3.23) and (3.31) that

$$
\begin{aligned}
\frac{\partial}{\partial s} F(s ; \lambda) & =-\frac{e^{\alpha \pi i}}{2 \pi i} \lim _{z \rightarrow-s}\left(X^{-1}(z) X^{\prime}(z)\right)_{21}=-\frac{e^{\alpha \pi i}}{2 \pi i s} \lim _{z \rightarrow-1}\left(T^{-1}(z) T^{\prime}(z)\right)_{21} \\
& =-\frac{e^{\alpha \pi i}}{2 \pi i s} \lim _{z \rightarrow-1}\left(e^{-\sqrt{s} g(z) \sigma_{3}} S^{-1}(z) S^{\prime}(z) e^{\sqrt{s} g(z) \sigma_{3}}\right)_{21},
\end{aligned}
$$

where the derivative is taken with respect to $z$ and the limit as $z \rightarrow-s$ is taken as $z \in$ region $V$ in Figure 2 .

When $z$ is close to -1 , it follows from (3.49) and (3.63) that

$$
S(z)=R(z) E_{-1}(z) \Phi_{\text {Bes }}^{(0)}\left(\frac{1}{4} s g^{2}(z)\right) \begin{cases}e^{\left(-\sqrt{s} g(z)+\frac{\alpha \pi i}{2}\right) \sigma_{3}}, & \text { for } \operatorname{Im} z>0 \\ e^{\left(-\sqrt{s} g(z)-\frac{\alpha \pi i}{2}\right) \sigma_{3}}, & \text { for } \operatorname{Im} z<0 .\end{cases}
$$

Thus, if $\operatorname{Im} z<0$, we obtain

$$
\begin{aligned}
e^{-\sqrt{s} g(z) \sigma_{3}} S^{-1}(z) S^{\prime}(z) e^{\sqrt{s} g(z) \sigma_{3}} & \\
=e^{\frac{\alpha \pi i}{2} \sigma_{3}}\left[\Phi_{\mathrm{Bes}}^{(0)}\right. & \left(\frac{s g^{2}(z)}{4}\right)^{-1} E_{-1}^{-1}(z) R^{-1}(z) R^{\prime}(z) E_{-1}(z) \Phi_{\mathrm{Bes}}^{(0)}\left(\frac{s g^{2}(z)}{4}\right) \\
+ & \Phi_{\mathrm{Bes}}^{(0)}\left(\frac{s g^{2}(z)}{4}\right)^{-1} E_{-1}^{-1}(z) E_{-1}^{\prime}(z) \Phi_{\mathrm{Bes}}^{(0)}\left(\frac{s g^{2}(z)}{4}\right) \\
& \left.+\Phi_{\mathrm{Bes}}^{(0)}\left(\frac{s g^{2}(z)}{4}\right)^{-1}\left[\Phi_{\mathrm{Bes}}^{(0)}\left(\frac{s g^{2}(z)}{4}\right)\right]^{\prime}-\sqrt{s} g^{\prime}(z) \sigma_{3}\right] e^{-\frac{\alpha \pi i}{2} \sigma_{3}} .
\end{aligned}
$$

Plugging the above expression into (3.69) implies that

$$
\begin{aligned}
& \frac{\partial}{\partial s} F(s ; \lambda) \\
& =\frac{-1}{2 \pi i s} \lim _{z \rightarrow-1}\left[\Phi_{\text {Bes }}^{(0)}\left(\frac{s g^{2}(z)}{4}\right)^{-1} E_{-1}^{-1}(z) R^{-1}(z) R^{\prime}(z) E_{-1}(z) \Phi_{\text {Bes }}^{(0)}\left(\frac{s g^{2}(z)}{4}\right)\right. \\
& +\Phi_{\text {Bes }}^{(0)}\left(\frac{s g^{2}(z)}{4}\right)^{-1} E_{-1}^{-1}(z) E_{-1}^{\prime}(z) \Phi_{\text {Bes }}^{(0)}\left(\frac{s g^{2}(z)}{4}\right) \\
& \left.+\Phi_{\text {Bes }}^{(0)}\left(\frac{s g^{2}(z)}{4}\right)^{-1}\left[\Phi_{\text {Bes }}^{(0)}\left(\frac{s g^{2}(z)}{4}\right)\right]^{\prime}\right]_{21} .
\end{aligned}
$$

We now evaluate the limits of the three terms on the right hand side of (3.71) one by one. We start with the last term. By (3.48), it is readily seen that

$$
\begin{aligned}
& {\left[\Phi_{\mathrm{Bes}}^{(0)}\left(\frac{s g^{2}(z)}{4}\right)^{-1}\left[\Phi_{\mathrm{Bes}}^{(0)}\left(\frac{s g^{2}(z)}{4}\right)\right]^{\prime}\right]_{21}} \\
& =\left.\left[-2 \pi i\left(I_{0}^{\prime}\left(2 \zeta^{1 / 2}\right)\right)^{2}+2 \pi i I_{0}\left(2 \zeta^{1 / 2}\right)\left(\frac{\zeta^{-1 / 2} I_{0}^{\prime}\left(2 \zeta^{1 / 2}\right)}{2}+I_{0}^{\prime \prime}\left(2 \zeta^{1 / 2}\right)\right)\right]\right|_{\zeta=s g^{2}(z) / 4} \\
& \quad \times\left(\frac{s g^{2}(z)}{4}\right)^{\prime}
\end{aligned}
$$


Recalling the following Taylor expansion as $\xi \rightarrow 0$ of the modified Bessel function $I_{0}$ (cf. [1]):

$$
I_{0}(\xi)=1+\frac{\xi^{2}}{4}+\cdots
$$

A direct calculation with the aid of (3.27), (3.72) and the above formula shows that

$$
\lim _{z \rightarrow-1}\left[\Phi_{\text {Bes }}^{(0)}\left(\frac{s g^{2}(z)}{4}\right)^{-1}\left[\Phi_{\operatorname{Bes}}^{(0)}\left(\frac{s g^{2}(z)}{4}\right)\right]^{\prime}\right]_{21}=\frac{i \pi}{2 s^{2 k}}\left(s^{k+1 / 2}-\lambda^{k} \eta_{0}\right)^{2} .
$$

Next, we observe that the $(2,1)$ entry of a matrix $P$ is invariant under the conjugation $\Phi_{\text {Bes }}^{(0)}\left(\frac{s g^{2}(z)}{4}\right)^{-1} P \Phi_{\text {Bes }}^{(0)}\left(\frac{s g^{2}(z)}{4}\right)$ as $z \rightarrow-1$. It is then immediate from (3.58), (3.61) and (3.62) that

$$
\begin{aligned}
\lim _{z \rightarrow-1}\left[\Phi_{\mathrm{Bes}}^{(0)}\left(\frac{s g^{2}(z)}{4}\right)^{-1} E_{-1}^{-1}(z) E_{-1}^{\prime}(z)\right. & \left.\Phi_{\mathrm{Bes}}^{(0)}\left(\frac{s g^{2}(z)}{4}\right)\right]_{21} \\
& =\left(\widetilde{E}^{-1}(-1) \widetilde{E}^{\prime}(-1)\right)_{21}=-i \pi \alpha \frac{s^{k+1 / 2}-\lambda^{k} \eta_{0}}{s^{k}} .
\end{aligned}
$$

Finally, for the first term, we see from (3.58) and (3.66) that

$$
\begin{aligned}
& E_{-1}^{-1}(z) R^{-1}(z) R^{\prime}(z) E_{-1}(z) \\
& \quad=\widetilde{E}_{-1}^{-1}(z)\left(I-\frac{R_{1}(z)}{s^{1 / 2}}+O(1 / s)\right)\left(\frac{R_{1}^{\prime}(z)}{s^{1 / 2}}+O(1 / s)\right) \widetilde{E}_{-1}(z),
\end{aligned}
$$

for large positive $s$. Hence,

$$
\begin{aligned}
& \lim _{z \rightarrow-1}\left[\Phi_{\operatorname{Bes}}^{(0)}\left(\frac{s g^{2}(z)}{4}\right)^{-1} E_{-1}^{-1}(z) R^{-1}(z) R^{\prime}(z) E_{-1}(z) \Phi_{\operatorname{Bes}}^{(0)}\left(\frac{s g^{2}(z)}{4}\right)\right]_{21} \\
& =\lim _{z \rightarrow-1}\left[E_{-1}^{-1}(z) R^{-1}(z) R^{\prime}(z) E_{-1}(z)\right]_{21}=\frac{1}{s^{1 / 2}} \lim _{z \rightarrow-1}\left[\widetilde{E}_{-1}^{-1}(z) R_{1}^{\prime}(z) \widetilde{E}_{-1}(z)\right]_{21}+O(1 / s) \\
& =\pi \frac{s^{k+1 / 2}-\lambda^{k} \eta_{0}}{s^{k+1 / 2}}\left(R_{1}^{\prime}(-1)\right)_{21}+O(1 / s)=i \pi\left(\frac{\alpha^{2}}{2}+\frac{3 \eta_{1}}{8} \frac{\lambda^{k}}{s^{k+1 / 2}-\lambda^{k} \eta_{0}}\right)+O(1 / s),
\end{aligned}
$$

where the first equality follows again from the fact that the $(2,1)$ entry of a matrix $P$ is invariant under the conjugation $\Phi_{\mathrm{Bes}}^{(0)}\left(\frac{s g^{2}(z)}{4}\right)^{-1} P \Phi_{\mathrm{Bes}}^{(0)}\left(\frac{s g^{2}(z)}{4}\right)$ as $z \rightarrow-1$, the second equality follows from (3.75), and the last two equalities follow directly from (3.61) and (3.68). A combination of (3.71), (3.73), (3.74) and (3.76) then gives us the following large $s$ asymptotics of $\frac{\partial}{\partial s} F(s ; \lambda)$ :

$$
\begin{aligned}
& \frac{\partial}{\partial s} F(s ; \lambda) \\
& =-\frac{1}{4 s^{2 k+1}}\left(s^{k+1 / 2}-\lambda^{k} \eta_{0}\right)^{2}+\alpha \frac{s^{k+1 / 2}-\lambda^{k} \eta_{0}}{2 s^{k+1}}-\frac{1}{2 s}\left(\frac{\alpha^{2}}{2}+\frac{3 \eta_{1}}{8} \frac{\lambda^{k}}{s^{k+1 / 2}-\lambda^{k} \eta_{0}}\right)+O\left(1 / s^{2}\right) \\
& =-\frac{1}{4}+\frac{\alpha}{2 s^{1 / 2}}-\frac{\alpha^{2}}{4 s}+O\left(1 / s^{\min (k+1 / 2,2)}\right) .
\end{aligned}
$$

\section{$4 \quad$ Large $s$ asymptotics of $\frac{\partial}{\partial \lambda} F(s ; \lambda)$}

To prove Theorem 2.1, it is also necessary to study the large $s$ asymptotics of $\frac{\partial}{\partial \lambda} F(s ; \lambda)$, which, together with the large $s$ asymptotics of $\frac{\partial}{\partial s} F(s ; \lambda)$, will gives us large $s$ asymptotics 
of $F(s ; \lambda)$ up to a constant term. It comes out that $\frac{\partial}{\partial \lambda} F(s ; \lambda)$ can not be related to the $\mathrm{RH}$ problem $X$ directly. Indeed, a straightforward calculation shows that, if $k=1$,

$$
\begin{aligned}
& \frac{d}{d \lambda} \widetilde{K}_{\mathrm{PIII}} \\
& =\frac{d}{d \lambda}\left(\frac{\mathbf{f}^{t}(u) \mathbf{h}(v)}{u-v}\right) \\
& =-\frac{e^{\alpha \pi i}}{2 \pi i u v}\left[q^{\prime}(\lambda)\left(\psi_{1}(u) \psi_{2}(v)+\psi_{2}(u) \psi_{1}(v)\right)-i r^{\prime}(\lambda) \psi_{2}(u) \psi_{2}(v)-i p^{\prime}(\lambda) \psi_{1}(u) \psi_{1}(v)\right],
\end{aligned}
$$

where the functions $p, q$ and $r$ are the same as those given in (1.10). The prefactor $1 /(u v)$ in the above formula will cause the difficulty.

To overcome this difficulty, we will consider a 'scaled' version of the Fredholm determinant. To this end, we first follow 3 and define

$$
\Phi(z ; \lambda)=\left(\begin{array}{cc}
1 & 0 \\
\lambda^{-1} r\left(\lambda^{2}\right) & 1
\end{array}\right) \lambda^{\frac{\sigma_{3}}{2}} e^{\frac{\pi i}{4} \sigma_{3}} \Psi\left(\lambda^{2} z ; \lambda^{2}\right),
$$

where $r$ is defined by (1.10). Next, for $x<0$, we set

$$
\widehat{\mathbf{f}}(x)=\Phi_{-}(x)\left(\begin{array}{c}
-e^{\frac{\alpha \pi i}{2}} \\
e^{-\frac{\alpha \pi i}{2}}
\end{array}\right)=\left(\begin{array}{c}
\widehat{f}_{1}(x) \\
\widehat{f}_{2}(x)
\end{array}\right), \quad \widehat{\mathbf{h}}(x)=-\frac{1}{2 \pi i} \Phi_{-}^{-t}(x)\left(\begin{array}{c}
e^{-\frac{\alpha \pi i}{2}} \\
e^{\frac{\alpha \pi i}{2}}
\end{array}\right)=\left(\begin{array}{c}
\widehat{h}_{1}(x) \\
\widehat{h}_{2}(x)
\end{array}\right) .
$$

Finally, let us denote by $\widehat{\mathcal{K}}_{\text {PIII }}$ the integral operator with kernel

$$
\chi_{[-s, 0]}(u) \widehat{K}_{\mathrm{PIII}}(u, v) \chi_{[-s, 0]}(v)=\chi_{[-s, 0]}(u) \frac{\widehat{\mathbf{f}}^{t}(u) \widehat{\mathbf{h}}(v)}{u-v} \chi_{[-s, 0]}(v)
$$

acting on the function space $L^{2}((-\infty, 0))$. By (4.2) and (4.3), it is readily seen that

$$
\chi_{[-s, 0]}(u) \widehat{K}_{\mathrm{PIII}}(u, v) \chi_{[-s, 0]}(v)=\chi_{[-s, 0]}(u) \frac{\mathbf{f}^{t}\left(\lambda^{2} u ; \lambda^{2}\right) \mathbf{h}\left(\lambda^{2} v ; \lambda^{2}\right)}{u-v} \chi_{[-s, 0]}(v) .
$$

Thus, a change of variable shows that

$$
\ln \operatorname{det}\left(I-\widehat{\mathcal{K}}_{\mathrm{PIII}}\right)=F\left(\lambda^{2} s ; \lambda^{2}\right) \text {. }
$$

Note that

$$
\frac{d}{d \lambda} \ln \operatorname{det}\left(I-\widehat{\mathcal{K}}_{\mathrm{PIII}}\right)=\frac{\partial}{\partial \lambda} F\left(\lambda^{2} s ; \lambda^{2}\right)=2\left(F_{s}\left(\lambda^{2} s ; \lambda^{2}\right) \lambda s+F_{\lambda}\left(\lambda^{2} s ; \lambda^{2}\right) \lambda\right) .
$$

Our strategy is the following. For large $s$, on one hand, as we will show later, the asymptotics of $\frac{d}{d \lambda} \ln \operatorname{det}\left(I-\widehat{\mathcal{K}}_{\text {PIII }}\right)$ can be obtained by performing a steepest descent analysis on a $\mathrm{RH}$ problem similar to $X$. On the other hand, the asymptotics of $F_{s}\left(\lambda^{2} s ; \lambda^{2}\right)$ is available in view of (3.77). The large $s$ asymptotics of $\frac{\partial}{\partial \lambda} F(s ; \lambda)$ can then be derived from (4.6) via change of variables $\lambda^{2} s \rightarrow s$ and $\lambda^{2} \rightarrow \lambda$.

In what follows, we first relate $\frac{d}{d \lambda} \ln \operatorname{det}\left(I-\widehat{\mathcal{K}}_{\text {PIII }}\right)$ to a $\mathrm{RH}$ problem following the same idea laid out in Section 3 , 


\subsection{A Riemann-Hilbert setting for $\frac{\partial}{\partial \lambda} F\left(\lambda^{2} s ; \lambda^{2}\right)$}

To proceed, we note from (4.2) and the RH problem for $\Psi$ that $\Phi$ satisfies the following $\mathrm{RH}$ problem (see also [3]).

RH problem 4.1. The function $\Phi(z)=\Phi(z ; \lambda)$ defined in (4.2) has the following properties:

(1) $\Phi(z)$ is defined and analytic in $\mathbb{C} \backslash\left\{\cup_{j=1}^{3} \Sigma_{j} \cup\{0\}\right\}$, where the contours $\Sigma_{j}$ are illustrated in Figure 1 .

(2) $\Phi$ satisfies the following jump conditions:

$$
\Phi_{+}(z)=\Phi_{-}(z)\left\{\begin{array}{l}
\left(\begin{array}{cc}
1 & 0 \\
e^{\alpha \pi i} & 1
\end{array}\right), \\
\left(\begin{array}{cc}
0 & 1 \\
-1 & 0
\end{array}\right), \quad z \in \Sigma_{1}, \\
\left(\begin{array}{cc}
1 & 0 \\
e^{-\alpha \pi i} & 1
\end{array}\right), \quad z \in \Sigma_{2},
\end{array}\right.
$$

(3) As $z \rightarrow \infty$,

$$
\begin{aligned}
\Phi(z ; \lambda)=\left(\begin{array}{cc}
1 & 0 \\
v(\lambda) & 1
\end{array}\right)\left(I+\frac{1}{z}\left(\begin{array}{cc}
w(\lambda) & v(\lambda) \\
l(\lambda) & -w(\lambda)
\end{array}\right)+\right. & \left.O\left(z^{-2}\right)\right) \\
& \times e^{\frac{\pi i}{4} \sigma_{3}} z^{-\frac{1}{4} \sigma_{3}} \frac{I+i \sigma_{1}}{\sqrt{2}} e^{\lambda z^{\frac{1}{2}} \sigma_{3}}
\end{aligned}
$$

where

$$
w(\lambda)=q\left(\lambda^{2}\right) / \lambda^{2}, \quad v(\lambda)=r\left(\lambda^{2}\right) / \lambda, \quad l(\lambda)=p\left(\lambda^{2}\right) / \lambda^{3} .
$$

(4) As $z \rightarrow 0$, there exists a matrix $\Phi_{0}(\lambda)$, independent of $z$, such that

$$
\Phi(z ; \lambda)=\Phi_{0}(\lambda)(I+O(z)) e^{-\left(-\frac{1}{z}\right)^{k} \sigma_{3}} z^{\frac{\alpha}{2} \sigma_{3}}\left\{\begin{array}{ccc}
I, & \\
\left(\begin{array}{cc}
1 & 0 \\
-e^{\alpha \pi i} & 1
\end{array}\right), & z \in \Omega_{2}, \\
\left(\begin{array}{cc}
1 & 0 \\
e^{-\alpha \pi i} & 1
\end{array}\right), & z \in \Omega_{3},
\end{array}\right.
$$

where the regions $\Omega_{i}, i=1,2,3$, are depicted in Figure 1 .

Furthermore, by [3. Equations (2.11) and (2.13)], one has

$$
\partial_{\lambda} \Phi=\left(\begin{array}{cc}
0 & 1 \\
z-u(\lambda) & 0
\end{array}\right) \Phi,
$$

where

$$
u(\lambda)=2 w(\lambda)-v^{\prime}(\lambda)-v(\lambda)^{2} .
$$


This, together with (4.3), implies that

$$
\frac{\partial \widehat{\mathbf{f}}}{\partial \lambda}(x)=\left(\begin{array}{cc}
0 & 1 \\
x-u(\lambda) & 0
\end{array}\right) \widehat{\mathbf{f}}(x), \quad \frac{\partial \widehat{\mathbf{h}}}{\partial \lambda}(x)=-\left(\begin{array}{cc}
0 & x-u(\lambda) \\
1 & 0
\end{array}\right) \widehat{\mathbf{h}}(x),
$$

and by (4.4),

$$
\frac{d}{d \lambda} \widehat{K}_{\mathrm{PIII}}=\widehat{\mathbf{f}}^{t}(u)\left(\begin{array}{ll}
0 & 1 \\
0 & 0
\end{array}\right) \widehat{\mathbf{h}}(v)=\widehat{f}_{1}(u) \widehat{h}_{2}(v)
$$

Thus, we obtain

$$
\frac{d}{d \lambda} \ln \operatorname{det}\left(I-\widehat{\mathcal{K}}_{\mathrm{PIII}}\right)=-\operatorname{tr}\left(\left(I-\widehat{\mathcal{K}}_{\mathrm{PIII}}\right)^{-1} \frac{d}{d \lambda} \widehat{\mathcal{K}}_{\mathrm{PIII}}\right)=-\int_{-s}^{0} \widehat{F}_{1}(v) \widehat{h}_{2}(v) d v,
$$

where, as in (3.6), $\widehat{\mathbf{F}}$ and $\widehat{\mathbf{H}}$ are defined as

$$
\widehat{\mathbf{F}}=\left(\begin{array}{c}
\widehat{F}_{1} \\
\widehat{F}_{2}
\end{array}\right):=\left(I-\widehat{\mathcal{K}}_{\mathrm{PIII}}\right)^{-1} \widehat{\mathbf{f}}, \quad \widehat{\mathbf{H}}=\left(\begin{array}{c}
\widehat{H}_{1} \\
\widehat{H}_{2}
\end{array}\right):=\left(I-\widehat{\mathcal{K}}_{\mathrm{PIII}}\right)^{-1} \widehat{\mathbf{h}} .
$$

To this end, we also set

$$
\widehat{Y}(z)=I-\int_{-s}^{0} \frac{\widehat{\mathbf{F}}(w) \widehat{\mathbf{h}}^{t}(w)}{w-z} d w
$$

It is then easily seen that

$$
\frac{d}{d \lambda} \ln \operatorname{det}\left(I-\widehat{\mathcal{K}}_{\mathrm{PIII}}\right)=-\left(\widehat{Y}_{\infty}\right)_{12},
$$

where $\widehat{Y}_{\infty}$ is the residue of $\widehat{Y}$ at infinity, i.e.,

$$
\widehat{Y}(z)=I+\frac{\widehat{Y}_{\infty}}{z}+O\left(z^{-2}\right), \quad z \rightarrow \infty
$$

Note that $\widehat{Y}$ also satisfies a RH problem similar to RH problem 3.1, and the only difference is the jump condition is replaced by

$$
\widehat{Y}_{+}(x)=\widehat{Y}_{-}(x)\left(I-2 \pi i \widehat{\mathbf{f}}(x) \widehat{\mathbf{h}}^{t}(x)\right), \quad x \in(-s, 0) .
$$

Thus, similar to the definition of $X$ given in (3.13), we define

$$
\widehat{X}(z)=\left\{\begin{array}{cc}
\widehat{Y}(z) \Phi(z), & \text { for } z \text { in region } \mathrm{I} \cup \mathrm{III} \cup \mathrm{IV}, \\
\widehat{Y}(z) \Phi(z)\left(\begin{array}{rr}
1 & 0 \\
e^{\alpha \pi i} & 1
\end{array}\right), & \text { for } z \text { in region II, } \\
\widehat{Y}(z) \Phi(z)\left(\begin{array}{rr}
1 & 0 \\
-e^{-\alpha \pi i} & 1
\end{array}\right), & \text { for } z \text { in region } \mathrm{V},
\end{array}\right.
$$

where the regions $\mathrm{I}-\mathrm{V}$ are shown in Figure 2, The following RH problem is straightforward to check from the above definition. 
RH problem 4.2. The function $\widehat{X}$ defined in (4.20) has the following properties:

(1) $\widehat{X}(z)$ is defined and analytic in $\mathbb{C} \backslash\left\{\cup_{j=1}^{4} \Gamma_{j}^{(s)} \cup\{-s\}\right\}$, where $\Gamma_{j}^{(s)}$ is defined in (3.14).

(2) $\widehat{X}$ satisfies the same jump conditions (3.15) as X.

(3) As $z \rightarrow \infty$,

$$
\widehat{X}(z)=\left(\begin{array}{cc}
1 & 0 \\
v(\lambda) & 1
\end{array}\right)\left(I+\frac{\widehat{X}_{\infty}}{z}+O\left(\frac{1}{z^{2}}\right)\right) e^{\frac{\pi i}{4} \sigma_{3}} z^{-\frac{1}{4} \sigma_{3}} \frac{I+i \sigma_{1}}{\sqrt{2}} e^{\lambda z^{\frac{1}{2}} \sigma_{3}},
$$

where

$$
\begin{aligned}
\widehat{X}_{\infty} & =\left(\begin{array}{cc}
1 & 0 \\
-v(\lambda) & 1
\end{array}\right) \widehat{Y}_{\infty}\left(\begin{array}{cc}
1 & 0 \\
v(\lambda) & 1
\end{array}\right)+\left(\begin{array}{cc}
w(\lambda) & v(\lambda) \\
l(\lambda) & -w(\lambda)
\end{array}\right) \\
& =\left(\begin{array}{cc}
* & \left(\widehat{Y}_{\infty}\right)_{12}+v(\lambda) \\
* & *
\end{array}\right) .
\end{aligned}
$$

(4) We have

$$
\widehat{X}(z)= \begin{cases}O(1)(I+O(z)) e^{\frac{(-1)^{k+1}}{z^{k}} \sigma_{3}} z^{\frac{\alpha}{2} \sigma_{3}}, & \text { as } z \rightarrow 0, \\ O(\ln (z+s)), & \text { as } z \rightarrow-s .\end{cases}
$$

The connection between $\widehat{X}$ and $\frac{\partial}{\partial \lambda} F\left(\lambda^{2} s ; \lambda^{2}\right)$ is stated in the following proposition, which follows directly from (4.17) and (4.22).

Proposition 4.3. We have

$$
\frac{\partial}{\partial \lambda} F\left(\lambda^{2} s ; \lambda^{2}\right)=\frac{d}{d \lambda} \ln \operatorname{det}\left(I-\widehat{\mathcal{K}}_{P I I I}\right)=v(\lambda)-\left(\widehat{X}_{\infty}\right)_{12} .
$$

\subsection{Asymptotic analysis of the Riemann-Hilbert problem for $\widehat{X}$}

In this section, we shall perform the Deift-Zhou steepest descent analysis to the RH problem for $\widehat{X}$ as $s \rightarrow+\infty$, which is similar to the analysis carried out in Section 3.2 ,

4.2.1 Transformations $\widehat{X} \rightarrow \widehat{T} \rightarrow \widehat{S}$

Define consecutive transformations

$$
\widehat{T}(z)=\widehat{X}(s z)
$$

and

$$
\widehat{S}(z)=s^{\frac{1}{4} \sigma_{3}} e^{-\frac{1}{4} \pi i \sigma_{3}}\left(\begin{array}{cc}
1 & 0 \\
s \widehat{\mathfrak{g}}_{1}-v(\lambda) & 1
\end{array}\right) \widehat{T}(z) e^{-\sqrt{s} \widehat{g}(z) \sigma_{3}},
$$

where

$$
\widehat{g}(z):=\sqrt{z+1}\left(\lambda+\frac{(-1)^{k+1}}{s^{k+1 / 2}} \sum_{j=1}^{k} \frac{c_{j}}{z^{j}}\right), \quad z \in \mathbb{C} \backslash(-\infty,-1],
$$


with the coefficients $c_{j}$ given in (3.28), and

$$
\widehat{\mathfrak{g}}_{1}=\frac{\lambda}{2}+\frac{(-1)^{k+1}}{s^{k+1 / 2}} c_{1} .
$$

From the definition of $\widehat{g}$ in (4.27), we have

$$
\widehat{g}_{+}(x)+\widehat{g}_{-}(x)=0, \quad x<-1,
$$

and

$$
\widehat{g}(z)= \begin{cases}\lambda \sqrt{z}+\widehat{\mathfrak{g}}_{1} z^{-1 / 2}+O\left(z^{-3 / 2}\right), & \text { as } z \rightarrow \infty, \\ \frac{(-1)^{k+1}}{s^{k+1 / 2} z^{k}}+O(1), & \text { as } z \rightarrow 0,\end{cases}
$$

where $\widehat{\mathfrak{g}}_{1}$ is given in (4.28).

It is then straightforward to check that $\widehat{S}$ satisfies the following RH problem.

RH problem 4.4. The function $\widehat{S}$ defined in (4.26) has the following properties:

(1) $\widehat{S}(z)$ is defined and analytic in $\mathbb{C} \backslash\left\{\cup_{j=1}^{4} \Gamma_{j}^{(1)} \cup\{-1\}\right\}$.

(2) $\widehat{S}$ satisfies the following jump conditions:

$$
\widehat{S}_{+}(z)=\widehat{S}_{-}(z) \begin{cases}\left(\begin{array}{cc}
e^{\alpha \pi i} & 0 \\
0 & e^{-\alpha \pi i}
\end{array}\right), & z \in \Gamma_{1}^{(1)}, \\
\left(\begin{array}{cc}
1 & 0 \\
e^{\alpha \pi i-2 \sqrt{s} \widehat{g}(z)} & 1
\end{array}\right), & z \in \Gamma_{2}^{(1)}, \\
\left(\begin{array}{cc}
0 & 1 \\
-1 & 0
\end{array}\right), & \\
\left(\begin{array}{cc}
1 & \\
\left(e^{-\alpha \pi i-2 \sqrt{s} \widehat{g}(z)}\right. & 1
\end{array}\right), & z \in \Gamma_{3}^{(1)},\end{cases}
$$

(3) As $z \rightarrow \infty$,

$$
\widehat{S}(z)=\left(I+\frac{\widehat{S}_{\infty}}{z}+O\left(\frac{1}{z^{2}}\right)\right) z^{-\frac{1}{4} \sigma_{3}} \frac{I+i \sigma_{1}}{\sqrt{2}},
$$

with

$$
\left(\widehat{S}_{\infty}\right)_{12}=-i \frac{\left(\widehat{X}_{\infty}\right)_{12}}{\sqrt{s}}+i \sqrt{s} \widehat{\mathfrak{g}}_{1},
$$

where $\widehat{\mathfrak{g}}_{1}$ is given in (4.28).

(4) We have

$$
\widehat{S}(z)= \begin{cases}O(1) z^{\frac{\alpha}{2} \sigma_{3}}, & \text { as } z \rightarrow 0, \\ O(\ln (z+1)), & \text { as } z \rightarrow-1 .\end{cases}
$$

By (4.27), again, one verifies that for sufficiently large positive $s$,

$$
\operatorname{Re} \widehat{g}(z)>0, \quad z \in \Gamma_{2}^{(1)} \cup \Gamma_{4}^{(1)} .
$$

Hence, the jump matrix of $\widehat{S}$ on $\Gamma_{2}^{(1)} \cup \Gamma_{4}^{(1)}$ tends to the identity matrix exponentially fast as $s \rightarrow+\infty$. This uniform convergence is not valid anymore for $z$ near -1 . 


\subsubsection{Outer parametrix, local parametrix near -1 and final transformation}

By ignoring the exponentially small terms in the jumps (4.30) and a neighborhood of -1 , we are led to the $\mathrm{RH}$ problem 3.7 for the outer parametrix $N$, whose solution is given by (3.38).

In a small, open neighborhood $D_{-1}$ near -1 with fixed radius, it is natural to consider a function $\widehat{P}_{-1}$ satisfying a $\mathrm{RH}$ problem as follows.

RH problem 4.5. We look for a $2 \times 2$ matrix-valued function $\widehat{P}_{-1}(z)$ satisfying

(1) $\widehat{P}_{-1}(z)$ is defined and analytic in $\overline{D_{-1}} \backslash\left\{\cup_{j=1}^{4} \Gamma_{j}^{(1)} \cup\{-1\}\right\}$.

(2) $\widehat{P}_{-1}(z)$ satisfies the same jump conditions (4.30) as $\widehat{S}$ for $z \in D_{-1} \cap\left\{\cup_{j=1}^{4} \Gamma_{j}^{(1)}\right\}$.

(3) As $s \rightarrow+\infty, \widehat{P}_{-1}$ matches $N(z)$ on the boundary $\partial D_{-1}$ of $D_{-1}$, i.e.,

$$
\widehat{P}_{-1}(z)=N(z)(I+o(1)), \quad z \in \partial D_{-1} .
$$

This $\mathrm{RH}$ problem is similar to $\mathrm{RH}$ problem 3.8 for $P_{-1}$, the only difference is that the function $g$ in the jump condition now is replaced by $\widehat{g}$. Hence, following the same arguments in Section 3.2.4, it is readily seen that $\widehat{P}_{-1}(z)$ is constructed in terms of the Bessel parametrix of order 0 as follows:

$$
\widehat{P}_{-1}(z)=\widehat{E}_{-1}(z) \Phi_{\text {Bes }}^{(0)}\left(\frac{1}{4} s \widehat{g}^{2}(z)\right) \begin{cases}e^{\left(-\sqrt{s} \widehat{g}(z)+\frac{\alpha \pi i}{2}\right) \sigma_{3}}, & \text { for } D_{-1} \cap\{z \mid \operatorname{Im} z>0\}, \\ e^{\left(-\sqrt{s} \widehat{g}(z)-\frac{\alpha \pi i}{2}\right) \sigma_{3}}, & \text { for } D_{-1} \cap\{z \mid \operatorname{Im} z<0\}\end{cases}
$$

where $\Phi_{\text {Bes }}^{(0)}$ is given in (3.48) with $\alpha=0$ and

$$
\widehat{E}_{-1}(z):=N(z) \begin{cases}e^{-\frac{\alpha \pi i}{2} \sigma_{3}} \frac{I-i \sigma_{1}}{\sqrt{2}}\left(\pi^{2} s \widehat{g}^{2}(z)\right)^{\frac{1}{4} \sigma_{3}}, & \text { for } \operatorname{Im} z>0 \\ e^{\frac{\alpha \pi i}{2} \sigma_{3}} \frac{I-i \sigma_{1}}{\sqrt{2}}\left(\pi^{2} s \widehat{g}^{2}(z)\right)^{\frac{1}{4} \sigma_{3}}, & \text { for } \operatorname{Im} z<0\end{cases}
$$

is analytic in $D_{-1}$.

The final transformation is defined by

$$
\widehat{R}(z)= \begin{cases}\widehat{S}(z) N(z)^{-1}, & \text { for } z \in \mathbb{C} \backslash D_{-1} \\ \widehat{S}(z) \widehat{P}_{-1}(z)^{-1}, & \text { for } z \in D_{-1}\end{cases}
$$

We then have the following RH problem for $\widehat{R}$.

RH problem 4.6. The $2 \times 2$ matrix-valued function $\widehat{R}(z)$ defined in (4.37) has the following properties:

(1) $\widehat{R}(z)$ is analytic in $\mathbb{C} \backslash \Sigma_{R}$, where the contour $\Sigma_{R}$ is shown in Figure 4.

(2) $\widehat{R}(z)$ satisfies the jump condition

$$
\widehat{R}_{+}(z)=\widehat{R}_{-}(z) J_{\widehat{R}}(z), \quad z \in \Sigma_{R},
$$

where

$$
J_{\widehat{R}}(z)= \begin{cases}\widehat{P}_{-1}(z) N(z)^{-1}, & \text { for } z \in \partial D_{-1} \\ N(z) J_{\widehat{S}}(z) N(z)^{-1}, & \text { for } z \in \Sigma_{R} \backslash \partial D_{-1}\end{cases}
$$


(3) As $z \rightarrow \infty$,

$$
\widehat{R}(z)=I+O(1 / z)
$$

The $\mathrm{RH}$ problem 4.6 is equivalent to the following singular integral equation:

$$
\widehat{R}(z)=I+\frac{1}{2 \pi i} \int_{\Sigma_{R}} \widehat{R}_{-}(w)\left(J_{\widehat{R}}(w)-I\right) \frac{d w}{w-z}, \quad z \in \mathbb{C} \backslash \Sigma_{R} .
$$

Furthermore, for large positive $s$, there exits some constant $c>0$ such that

$$
J_{\widehat{R}}(z)=I+O\left(e^{-c \sqrt{s}}\right), \quad z \in \Sigma_{R} \backslash \overline{D_{-1}} ;
$$

while for $z \in \partial D_{-1}$, as in (3.56), we have

$$
J_{\widehat{R}}(z)=I+\frac{1}{s^{1 / 2}}\left(\begin{array}{cc}
1 & 0 \\
i \alpha & 1
\end{array}\right) \widehat{J}_{1}(z)\left(\begin{array}{cc}
1 & 0 \\
-i \alpha & 1
\end{array}\right)+O\left(s^{-1}\right),
$$

where

$$
\widehat{J}_{1}(z)=\left(\begin{array}{cc}
\frac{\rho(z)^{-2}-\rho(z)^{2}}{8 \widehat{g}(z)} & \frac{-i\left(\left(\rho(z)+\rho(z)^{-1}\right)^{2}-3\right)}{8 \sqrt{z+1} \widehat{g}(z)} \\
\frac{-i\left(\left(\rho(z)+\rho(z)^{-1}\right)^{2}-1\right) \sqrt{z+1}}{8 \widehat{g}(z)} & \frac{\rho(z)^{2}-\rho(z)^{-2}}{8 \widehat{g}(z)}
\end{array}\right)
$$

with $\rho(z)$ defined in (3.55). Hence, as in (3.66),$\widehat{R}(z)$ has the following asymptotic expansion

$$
\widehat{R}(z)=I+\frac{1}{s^{1 / 2}}\left(\begin{array}{cc}
1 & 0 \\
\alpha i & 1
\end{array}\right) \widehat{R}_{1}(z)\left(\begin{array}{cc}
1 & 0 \\
-\alpha i & 1
\end{array}\right)+O\left(s^{-1}\right),
$$

where

$$
\widehat{R}_{1}(z)=\frac{1}{2 \pi i} \oint_{\partial D_{-1}} \frac{\widehat{J}_{1}(\zeta)}{\zeta-z} d \zeta= \begin{cases}\left(\begin{array}{cc}
0 & \frac{1}{8 i(z+1)\left(\lambda-\frac{\eta_{0}}{s^{k+1 / 2}}\right)} \\
0 & 0
\end{array}\right)-\widehat{J}_{1}(z), & \text { for } z \in D_{-1}, \\
\left(\begin{array}{cc}
0 & \frac{1}{8 i(z+1)\left(\lambda-\frac{\eta_{0}}{s^{k+1 / 2}}\right)} \\
0 & 0
\end{array}\right), & \text { for } z \in \mathbb{C} \backslash D_{-1},\end{cases}
$$

and where $\eta_{0}$ is given in (3.42).

\subsection{Large $s$ asymptotics of $\frac{\partial}{\partial \lambda} F(s ; \lambda)$}

By (4.24), (4.28) and (4.32), it follows that

$$
\frac{\partial}{\partial \lambda} F\left(\lambda^{2} s ; \lambda^{2}\right)=\frac{d}{d \lambda} \ln \operatorname{det}\left(I-\widehat{\mathcal{K}}_{\mathrm{PIII}}\right)=v(\lambda)-i \sqrt{s}\left(\widehat{S}_{\infty}\right)_{12}-\frac{\lambda}{2} s+O\left(s^{-1 / 2}\right) .
$$

To find the large $s$ asymptotics of $\left(\widehat{S}_{\infty}\right)_{12}$, we first observe from (4.37) that

$$
\widehat{S}(z)=\widehat{R}(z) N(z), \quad z \in \mathbb{C} \backslash D_{-1} .
$$


Next, let $z \rightarrow \infty$, on one hand, it is readily seen from (3.38) that

$$
N(z)=\left(I+\frac{N_{\infty}}{z}+O\left(z^{-2}\right)\right) z^{-\frac{1}{4} \sigma_{3}} \frac{I+i \sigma_{1}}{\sqrt{2}}
$$

where

$$
N_{\infty}=\left(\begin{array}{ll}
* & i \alpha \\
* & *
\end{array}\right) .
$$

On the other hand, we see from (4.39) that

$$
\widehat{R}(z)=I+\frac{\widehat{R}_{\infty}}{z}+O\left(z^{-2}\right),
$$

where

$$
\widehat{R}_{\infty}=\frac{i}{2 \pi} \int_{\Sigma_{R}} \widehat{R}_{-}(w)\left(J_{\widehat{R}}(w)-I\right) d w .
$$

If we further let $s \rightarrow+\infty$, this, together with (4.40), (4.41) and (4.43), implies that

$$
\begin{aligned}
\widehat{R}_{\infty} & =\frac{i}{2 \pi s^{1 / 2}} \oint_{\partial D_{-1}}\left(\begin{array}{cc}
1 & 0 \\
i \alpha & 1
\end{array}\right) \widehat{J}_{1}(w)\left(\begin{array}{cc}
1 & 0 \\
-i \alpha & 1
\end{array}\right) d w+O\left(s^{-1}\right) \\
& =\frac{1}{s^{1 / 2}}\left(\begin{array}{cc}
* & -\frac{i}{8} \frac{1}{\lambda-\frac{\eta_{0}}{s^{k+1 / 2}}} \\
* & *
\end{array}\right)+O\left(s^{-1}\right) .
\end{aligned}
$$

Finally, inserting (4.47), (4.48) and (4.49) into (4.46), it is easily seen from (4.31) that

$$
\left(\widehat{S}_{\infty}\right)_{12}=i \alpha-\frac{i}{8 s^{1 / 2}} \frac{1}{\lambda-\frac{\eta_{0}}{s^{k+1 / 2}}}+O(1 / s)
$$

Substituting the above asymptotics into (4.45), we arrive at

$$
\frac{\partial}{\partial \lambda} F\left(\lambda^{2} s ; \lambda^{2}\right)=-\frac{\lambda s}{2}+\alpha s^{1 / 2}+v(\lambda)-\frac{1}{8 \lambda}+O\left(s^{-1 / 2}\right), \quad s \rightarrow+\infty .
$$

Recall now the identity (4.6). On account of (3.77), it is readily seen that

$$
F_{s}\left(\lambda^{2} s ; \lambda^{2}\right) \lambda s=-\frac{\lambda s}{4}+\frac{\alpha}{2} s^{1 / 2}-\frac{\alpha^{2}}{4 \lambda}+O\left(s^{-1 / 2}\right) .
$$

A combination of (4.6), (4.50) and (4.51) gives us

$$
F_{\lambda}\left(\lambda^{2} s ; \lambda^{2}\right)=\frac{v(\lambda)}{2 \lambda}+\frac{\alpha^{2}}{4 \lambda^{2}}-\frac{1}{16 \lambda^{2}}+O\left(s^{-1 / 2}\right), \quad s \rightarrow+\infty,
$$

or equivalently, by (4.9),

$$
F_{\lambda}(s ; \lambda)=\frac{1}{2 \lambda}\left(r(\lambda)+\frac{\alpha^{2}}{2}-\frac{1}{8}\right)+O\left(s^{-1 / 2}\right), \quad s \rightarrow+\infty .
$$

We are now ready to prove Theorem 2.1 


\section{Proof of Theorem 2.1}

From (3.77) and (4.53), it is immediate that

$$
\begin{aligned}
\ln \operatorname{det}\left(I-\widetilde{\mathcal{K}}_{\text {PIII }}\right)=-\frac{1}{4} s & +\alpha s^{1 / 2}-\frac{\alpha^{2}}{4} \ln s \\
& +\int_{0}^{\lambda} \frac{1}{2 t}\left(r(t)+\frac{\alpha^{2}}{2}-\frac{1}{8}\right) d t+\widehat{\tau}_{\alpha}+O\left(s^{-1 / 2}\right), \quad s \rightarrow+\infty,
\end{aligned}
$$

where $\widehat{\tau}_{\alpha}$ is a constant independent of $\lambda$ and $s$. From the asymptotics of $r(\lambda)$ given in (1.22), we have that the integral in the above formula is well-defined, and tends to 0 as $\lambda \rightarrow 0^{+}$.

By (1.17), we have

$$
\lim _{\lambda \rightarrow 0^{+}} \ln \operatorname{det}\left(I-\widetilde{\mathcal{K}}_{\mathrm{PIII}}\right)=\ln \operatorname{det}\left(I-\mathcal{K}_{\mathrm{Bes}}\right),
$$

where $\mathcal{K}_{\text {Bes }}$ is the integral operator with kernel $\chi_{[0, s]}(u) K_{\text {Bes }}(u, v) \chi_{[0, s]}(v)$ acting on the function space $L^{2}((0, \infty))$. We mention that (5.2) can also be seen from the consistency of (2.12) with the logarithm of (1.26), as shown in the Appendix below. Letting $s \rightarrow+\infty$ on both side of the above formula, it then follows from the large $s$ asymptotics of $\operatorname{det}\left(I-\mathcal{K}_{\text {Bes }}\right)$ in (1.29) and (5.1) that

$$
\widehat{\tau}_{\alpha}=\tau_{\alpha}=\ln \left(\frac{G(1+\alpha)}{(2 \pi)^{\alpha / 2}}\right) .
$$

This completes the proof of Theorem 2.1.

\section{Derivation of the coupled Painlevé III system}

In this section, we derive the coupled Painlevé III system (1.19) which is relevant to the gap probability.

\subsection{Riemann-Hilbert problem for $M$}

We start with a matrix-valued function $M(z ; \lambda, s)$, which is defined via $X(z ; \lambda, s)$ in (3.13) in the following way:

$$
M(z ; \lambda, s)=\left(\begin{array}{cc}
1 & 0 \\
a(\lambda ; s) & 1
\end{array}\right) \lambda^{\frac{\sigma_{3}}{2}} e^{\frac{\pi i}{4} \sigma_{3}} X\left(\lambda^{2} z ; \lambda^{2}, \lambda^{2} s\right) .
$$

From (3.21), it is readily seen that $M$ is related to the gap probability as follows:

$$
F_{s}\left(\lambda^{2} s ; \lambda^{2}\right)=-\frac{e^{\alpha \pi i}}{2 \pi i \lambda^{2}} \lim _{z \rightarrow-s}\left(M_{-}^{-1}(z) M_{-}^{\prime}(z)\right)_{21}, \quad \lambda>0
$$

where the limit is taken as $z$ tends to $-s$ in region $\mathrm{V}$. This will be our starting point in the proof of Theorem 2.5.

In view of the $\mathrm{RH}$ problem for $X$ stated in Proposition 3.2, we have

RH problem 6.1. The function $M$ defined in (6.1) has the following properties:

(1) $M(z)$ is analytic in $\mathbb{C} \backslash\left\{\cup_{j=1}^{4} \Gamma_{j}^{(s)} \cup\{-s\}\right\}$, where $\Gamma_{j}^{(s)}$ is defined in (3.14), as illustrated in solid lines in Figure $\mathbf{Q}$. 
(2) $M(z)$ satisfies the same jump conditions (3.15) as X.

(3) As $z \rightarrow \infty$,

$$
M(z)=\left(\begin{array}{cc}
1 & 0 \\
a(\lambda ; s) & 1
\end{array}\right)\left(I+\frac{M_{\infty}}{z}+O\left(\frac{1}{z^{2}}\right)\right) e^{\frac{\pi i}{4} \sigma_{3}} z^{-\frac{1}{4} \sigma_{3}} \frac{I+i \sigma_{1}}{\sqrt{2}} e^{\lambda z^{\frac{1}{2}} \sigma_{3}},
$$

where

$$
a(\lambda ; s)=\left(M_{\infty}\right)_{12}
$$

(4) $A s z \rightarrow 0$,

$$
M(z)=M^{(0)}(z) e^{\frac{(-1)^{k+1}}{z^{k}} \sigma_{3}} z^{\frac{\alpha}{2} \sigma_{3}}
$$

where $M^{(0)}(z)$ is analytic at $z=0$.

(5) $A s z \rightarrow-s$,

$$
M(z)=M^{(s)}(z)\left(I+\frac{1}{2 \pi i} \log (z+s) \sigma_{+}\right) E^{(s)}(z),
$$

where $M^{(s)}(z)$ is analytic at $z=-s$, i.e., there exist matrices $M_{j}^{(s)}, j=0,1,2, \ldots$, such that

$$
M^{(s)}(z)=M_{0}^{(s)}\left(I+\sum_{j=1}^{\infty} M_{j}^{(s)}(z+s)^{k}\right)
$$

$\sigma_{+}=\left(\begin{array}{ll}0 & 1 \\ 0 & 0\end{array}\right)$, and $E^{(s)}(z)$ is a piecewise constant matrix-valued function defined by

$$
E^{(s)}(z)=\left\{\begin{array}{cc}
e^{\frac{\alpha \pi i}{2} \sigma_{3}}, & z \in I I, \\
\left(\begin{array}{cc}
e^{\frac{\alpha \pi i}{2}} & 0 \\
-e^{\frac{\alpha \pi i}{2}} & e^{-\frac{\alpha \pi i}{2}}
\end{array}\right), & z \in I I I, \\
\left(\begin{array}{cc}
e^{-\frac{\alpha \pi i}{2}} & 0 \\
e^{-\frac{\alpha \pi i}{2}} & e^{\frac{\alpha \pi i}{2}}
\end{array}\right), & z \in I V \\
e^{-\frac{\alpha \pi i}{2} \sigma_{3}}, & z \in V,
\end{array}\right.
$$

with the regions $I I-V$ being depicted in Figure 2 .

The analyticity of the functions $M^{(0)}(z)$ and $M^{(s)}(z)$ follows directly from the jump condition for $M(z)$, and the definition of the piecewise constant matrix-valued function $E^{(s)}(z)$ in (6.8) (for $M^{(s)}(z)$ ).

We now show that the $\mathrm{RH}$ problem for $M$ is uniquely solvable through a standard vanishing lemma argument.

Lemma 6.2 (Vanishing Lemma). Let $\widehat{M}$ satisfies the 'homogeneous' version of the RH problem for $M$, i.e., $\widehat{M}$ satisfies $R H$ problem 6.1 but with the asymptotic behavior near infinity replaced by

$$
\widehat{M}(z)=O(1 / z) e^{\frac{\pi i}{4} \sigma_{3}} z^{-\frac{1}{4} \sigma_{3}} \frac{I+i \sigma_{1}}{\sqrt{2}} e^{\lambda z^{\frac{1}{2}} \sigma_{3}} .
$$


Then, this $R H$ problem has only the trivial solution for the parameters $\alpha>-1, s \geq 0$ and $\lambda>0$, that is,

$$
\widehat{M}(z) \equiv 0 .
$$

Proof. The case for $s=0$ and $k=1$ is proved in [44, Lemma 1]. For general $s>0$ and $k \in \mathbb{N}$, the proof is similar. We omit the details but point out that one important observation in the proof is the complex conjugation relation satisfied by jump matrices (3.15):

$$
\overline{\left(J_{1}^{(s)}\right)_{11}}=\left(J_{1}^{(s)}\right)_{22}, \quad \overline{\left(J_{2}^{(s)}\right)_{12}}=\left(J_{4}^{(s)}\right)_{12},
$$

where $J_{i}^{(s)}, i=1,2,3,4$, stands for the jump matrix of $M$ restricted on the contour $\Gamma_{i}^{(s)}$.

By means of the vanishing lemma 6.2 [19, 24, 46], the following theorem is immediate.

Theorem 6.3. Assume that $\alpha>-1, s \geq 0$ and $\lambda>0$. Then, the RH problem 6.1 for $M(z)$ is uniquely solvable.

\subsection{Lax pair equations and a coupled Painlevé III system}

We next obtain linear differential equations for $M$ with respect to $z, \lambda$ and $s$. This system of differential equations has a Lax pair form and the compatibility condition of the Lax pair will then lead to the coupled Painlevé III system (2.4).

Proposition 6.4. We have the following differential equations for $M=M(z ; \lambda, s)$ :

$$
\begin{aligned}
& \frac{\partial M}{\partial z}=\left(\sum_{j=1}^{k+1} \frac{A_{j}}{z^{j}}+\frac{A_{0}}{z+s}+\frac{\lambda}{2} \sigma_{-}\right) M, \\
& \frac{\partial M}{\partial \lambda}=\left(\begin{array}{cc}
0 & 1 \\
z+2 a^{\prime} & 0
\end{array}\right) M, \\
& \frac{\partial M}{\partial s}=\left(\frac{\partial a}{\partial s} \sigma_{-}+\frac{A_{0}}{z+s}\right) M,
\end{aligned}
$$

where $\sigma_{-}=\left(\begin{array}{ll}0 & 0 \\ 1 & 0\end{array}\right),^{\prime}=\frac{d}{d \lambda}$

$$
A_{j}=\left(\begin{array}{cc}
-\frac{b_{j}^{\prime}}{2} & b_{j} \\
-\frac{b_{j}^{\prime \prime}}{2}+2 a^{\prime} b_{j}+b_{j+1} & \frac{b_{j}^{\prime}}{2}
\end{array}\right) \quad \text { for } 1 \leq j \leq k+1
$$

and

$$
A_{0}=\left(\begin{array}{cc}
-\frac{1}{4}+\frac{b_{1}^{\prime}}{2} & \frac{\lambda}{2}-b_{1} \\
\frac{b_{1}^{\prime \prime}}{2}+\left(2 a^{\prime}-s\right)\left(\frac{\lambda}{2}-b_{1}\right) & \frac{1}{4}-\frac{b_{1}^{\prime}}{2}
\end{array}\right) .
$$

Moreover, the functions $a(\lambda ; s), b_{1}(\lambda ; s), \ldots, b_{k+1}(\lambda ; s)$ in (6.13) and (6.14) satisfy the coupled Painlevé III system (2.4). 
Proof. Since the jumps in the RH problem for $M(z)$ are $z, \lambda, s$-independent matrices, we have that the functions

$$
A(z ; \lambda, s):=\frac{\partial M}{\partial z} M^{-1}, \quad B(z ; \lambda, s):=\frac{\partial M}{\partial \lambda} M^{-1}, \quad C(z ; \lambda, s):=\frac{\partial M}{\partial s} M^{-1}
$$

are meromorphic functions with possible isolated singular points at $z=-s$ and $z=0$. From the asymptotic behaviors of $M$ near $z=\infty, z=-s$ and $z=0$ as given in (6.3)-(6.6), it follows that

$$
\begin{aligned}
& A(z ; \lambda, s)=\sum_{j=1}^{k+1} \frac{A_{j}}{z^{j}}+\frac{A_{0}}{z+s}+\frac{\lambda}{2} \sigma_{-}, \\
& B(z ; \lambda, s)=\left(\begin{array}{cc}
0 & 1 \\
z+a^{\prime}+a^{2}-2\left(M_{\infty}\right)_{11} & 0
\end{array}\right), \\
& C(z ; \lambda, s)=\frac{\partial a}{\partial s} \sigma_{-}+\frac{C_{1}}{z+s},
\end{aligned}
$$

for some $z$-independent matrices $A_{j}, j=0, \ldots, k+1$, and $C_{1}$. By (6.7), we further obtain

$$
A_{0}=C_{1}=\frac{1}{2 \pi i} M_{0}^{(s)} \sigma_{+}\left(M_{0}^{(s)}\right)^{-1}
$$

where $M_{0}^{(s)}$ is the leading term of the expansion in (6.7). The regularity of $B(z ; \lambda, s)$ in (6.17) also implies the $1 / z$ term in the expansion of $B(z ; \lambda, s)$ as $z \rightarrow \infty$ must vanish. Hence, by the definition of $B(z ; \lambda, s)$ given in (6.15) and (6.3), we find that

$$
a^{\prime}-a^{2}+2\left(M_{\infty}\right)_{11}=0 .
$$

This, together with (6.17), gives us (6.11).

Next, we show that the matrices $A_{j}, j=0, \ldots, k+1$, are given by (6.13) and (6.14). To proceed, we note that the compatibility condition

$$
\frac{\partial^{2} M}{\partial z \partial \lambda}=\frac{\partial^{2} M}{\partial \lambda \partial z}
$$

for the differential equations (6.10) and (6.11) is the zero curvature relation

$$
\frac{\partial A}{\partial \lambda}-\frac{\partial B}{\partial z}+[A, B]=0,
$$

where $[L, K]=L K-K L$ stands for the standard commutator of two matrices. Substituting (6.16) and (6.17) into the above formula, we obtain

$$
A_{j}^{\prime}+\left[A_{j}, B_{0}\right]+\left[A_{j+1}, \sigma_{-}\right]=0, \quad 1 \leq j \leq k+1,
$$

where $B_{0}=\left(\begin{array}{cc}0 & 1 \\ 2 a^{\prime} & 0\end{array}\right)$ and $A_{k+2}=\mathbf{0}_{2 \times 2}$. Thus, if we set

$$
b_{j}=\left(A_{j}\right)_{12},
$$

and rewrite (6.22) in an entrywise manner, it follows that

$$
\begin{aligned}
\left(A_{j}\right)_{11}^{\prime}-\left(A_{j}\right)_{21}+2 a^{\prime} b_{j}+b_{j+1} & =0 \\
\lambda b_{j}^{\prime}+2\left(A_{j}\right)_{11} & =0 \\
\left(A_{j}\right)_{21}^{\prime}-4 a^{\prime}\left(A_{j}\right)_{11}+b_{j+1}^{\prime} & =0 .
\end{aligned}
$$


Since $\operatorname{det} M \equiv 1$, we have

$$
\operatorname{tr} A=0 .
$$

A combination of (6.23), (6.24) and the above formula gives us the expression of $A_{j}$ in (6.13).

To see the expression of $A_{0}$ in (6.14), we note that

$$
\left(A_{0}+A_{1}\right)_{12}=\lambda / 2 .
$$

This follows from inserting (6.3) into the definition of $A(z ; \lambda, s)$ and comparing the coefficients of $O(1 / z)$ term in both sides as $z \rightarrow \infty$. Thus, $\left(A_{0}\right)_{12}=\lambda / 2-b_{1}$. The expression (6.14) as well as the relation (2.6) then follows from the compatibility condition

$$
\frac{\partial^{2} M}{\partial \lambda \partial s}=\frac{\partial^{2} M}{\partial s \partial \lambda}
$$

for the differential equations (6.11) and (6.12).

Finally, we derive the coupled Painlevé system (2.4) by evaluating the determinant of $A$. By (6.19), it is easily seen that

$$
\operatorname{det} A_{0}=0 .
$$

This, together with (6.14), gives us the first equation in (2.4). Moreover, from the asymptotic behavior of $M$ near the origin given in (6.5), we have

$$
A(z ; \lambda, s)=\frac{\partial M^{(0)}}{\partial z}\left(M^{(0)}\right)^{-1}+\left(\frac{(-1)^{k} k}{z^{k+1}}+\frac{\alpha}{2 z}\right) M^{(0)} \sigma_{3}\left(M^{(0)}\right)^{-1},
$$

which particularly implies that, as $z \rightarrow 0$,

$$
\operatorname{det} A(z ; \lambda, s)=-\frac{k^{2}}{z^{2 k+2}}+\frac{(-1)^{k+1} \alpha k}{z^{k+2}}+O\left(\frac{1}{z^{k+1}}\right) .
$$

The next $k+1$ equations in (2.4) then follow from the above formula, (6.13), (6.14) and (6.16).

This completes the proof of Proposition 6.4.

Remark 6.5. From (6.23)-(6.25), it follows that each $b_{j}$ also satisfies a third order ODE. We will not use this fact in this paper.

Remark 6.6. When $s=0$, the two singular points $z=0$ and $z=-s$ of $M(z ; \lambda, s)$ coalesce. Then, the coefficient $A_{0}$ in (6.10) and (6.12) is a zero matrix, which means $b_{1}=\lambda / 2$.

\section{Asymptotic analysis of the Riemann-Hilbert problem for $M$}

In this section, we will perform the Deift-Zhou steepest descent analysis to the RH problem for $M$ as $\lambda \rightarrow 0^{+}$, which will then lead to the asymptotics of $a(\lambda ; s)$ and $b_{1}(\lambda ; s)$ in (2.10) and (2.11), respectively.

\section{1 $M \rightarrow U$ : Rescaling and normalization}

Define

$$
U(z)=e^{-\frac{\pi i}{4} \sigma_{3}} \lambda^{-\frac{1}{2} \sigma_{3}}\left(\begin{array}{cc}
1 & 0 \\
-a(\lambda ; s) & 1
\end{array}\right) M\left(z / \lambda^{2}\right) .
$$

Then, it is straightforward to check that $U$ satisfies the following $\mathrm{RH}$ problem. 
RH problem 7.1. The function $U$ defined in (7.1) has the following properties:

(1) $U(z)$ is defined and analytic in $\mathbb{C} \backslash\left\{\cup_{j=1}^{4} \Gamma_{j}^{\left(\lambda^{2} s\right)} \cup\left\{-\lambda^{2} s\right\}\right\}$, where the definition of $\Gamma_{j}^{(s)}$ is given in (3.14).

(2) U satisfies the following jump conditions:

$$
U_{+}(z)=U_{-}(z) \begin{cases}\left(\begin{array}{cc}
e^{\alpha \pi i} & 0 \\
0 & e^{-\alpha \pi i}
\end{array}\right), & z \in \Gamma_{1}^{\left(\lambda^{2} s\right)}, \\
\left(\begin{array}{cc}
1 & 0 \\
e^{\alpha \pi i} & 1
\end{array}\right), & z \in \Gamma_{2}^{\left(\lambda^{2} s\right)}, \\
\left(\begin{array}{cc}
0 & 1 \\
-1 & 0
\end{array}\right), & z \in \Gamma_{3}^{\left(\lambda^{2} s\right)}, \\
\left(\begin{array}{cc}
1 & 0 \\
e^{-\alpha \pi i} & 1
\end{array}\right), & z \in \Gamma_{4}^{\left(\lambda^{2} s\right)} .\end{cases}
$$

(3) As $z \rightarrow \infty$,

$$
U(z)=\left(I+\frac{U_{\infty}}{z}+O\left(\frac{1}{z^{2}}\right)\right) z^{-\frac{1}{4} \sigma_{3}} \frac{I+i \sigma_{1}}{\sqrt{2}} e^{\sqrt{z} \sigma_{3}} .
$$

(4) As $z \rightarrow 0$,

$$
U(z)=O(1) e^{\frac{(-1)^{k+1} \lambda^{2 k}}{z^{k}} \sigma_{3}} z^{\frac{\alpha}{2} \sigma_{3}}
$$

(5) $A s z \rightarrow-\lambda^{2} s$,

$$
U(z)=O(1)\left(I+\frac{1}{2 \pi i} \log \left(z+\lambda^{2} s\right) \sigma_{+}\right) E^{\left(\lambda^{2} s\right)}(z),
$$

where the function $E^{\left(\lambda^{2} s\right)}(z)$ is a piecewise constant matrix-valued function defined by

$$
E^{\left(\lambda^{2} s\right)}(z)=\left\{\begin{array}{cc}
e^{\frac{\alpha \pi i}{2} \sigma_{3},} & \arg \left(z+\lambda^{2} s\right) \in\left(0, \frac{2 \pi}{3}\right), \\
\left(\begin{array}{cc}
e^{\frac{\alpha \pi i}{2}} & 0 \\
-e^{\frac{\alpha \pi i}{2}} & e^{-\frac{\alpha \pi i}{2}}
\end{array}\right), & \arg \left(z+\lambda^{2} s\right) \in\left(\frac{2 \pi}{3}, \pi\right), \\
\left(\begin{array}{cc}
e^{-\frac{\alpha \pi i}{2}} & 0 \\
e^{-\frac{\alpha \pi i}{2}} & e^{\frac{\alpha \pi i}{2}}
\end{array}\right), & \arg \left(z+\lambda^{2} s\right) \in\left(-\pi,-\frac{2 \pi}{3}\right), \\
e^{-\frac{\alpha \pi i}{2} \sigma_{3}}, & \arg \left(z+\lambda^{2} s\right) \in\left(-\frac{2 \pi}{3}, 0\right) .
\end{array}\right.
$$

\subsection{Outer parametrix}

As $\lambda \rightarrow 0^{+}$, the jump contour $\Gamma_{1}^{\left(\lambda^{2} s\right)}$ disappears, it is then natural to consider the following $\mathrm{RH}$ problem.

RH problem 7.2. We look for a $2 \times 2$ matrix-valued function $\Upsilon(z)$ satisfying

(1) $\Upsilon(z)$ is defined and analytic in $\mathbb{C} \backslash\left\{\cup_{j=1}^{3} \Sigma_{j} \cup\{0\}\right\}$, where the contours $\Sigma_{j}$ are illustrated in Figure 1. 
(2) $\Upsilon(z)$ satisfies the following jump conditions:

$$
\Upsilon_{+}(z)=\Upsilon_{-}(z)\left\{\begin{array}{l}
\left(\begin{array}{cc}
1 & 0 \\
e^{\pi i \alpha} & 1
\end{array}\right), \quad z \in \Sigma_{1}, \\
\left(\begin{array}{cc}
0 & 1 \\
-1 & 0
\end{array}\right), \\
\left(\begin{array}{cc}
1 & 0 \\
e^{-\pi i \alpha} & 1
\end{array}\right), \quad z \in \Sigma_{2},
\end{array}\right.
$$

(3) As $z \rightarrow \infty$, the behavior of $\Upsilon$ is the same as that of $U$ given in (7.3).

As shown in [3, Sec. 5.1] and [44, Sec. 5.1], the solution to RH problem[.2 can be constructed explicitly with the aid of the Bessel parametrix $\Phi_{\text {Bes }}^{(\alpha)}$ of order $\alpha(3.48)$ in the following way:

$$
\Upsilon(z)=\left(I+\frac{i}{8}\left(4 \alpha^{2}+3\right) \sigma_{-}\right) \pi^{\frac{\sigma_{3}}{2}} \Phi_{\mathrm{Bes}}^{(\alpha)}(z / 4) .
$$

As a consequence, the behavior of $\Upsilon(z)$ as $z \rightarrow \infty$ in (7.3) can be refined to be

$$
\begin{aligned}
\Upsilon(z)=\left[I+\frac{4 \alpha^{2}-1}{128 z}\left(\begin{array}{cc}
4 \alpha^{2}-9 & 16 i \\
\frac{i}{12}\left(4 \alpha^{2}-9\right)\left(4 \alpha^{2}-13\right) & 9-4 \alpha^{2}
\end{array}\right)+\right. & \left.O\left(z^{-\frac{3}{2}}\right)\right] \\
& \times z^{-\frac{1}{4} \sigma_{3}} \frac{I+i \sigma_{1}}{\sqrt{2}} e^{\sqrt{z} \sigma_{3}}
\end{aligned}
$$

Moreover, from (3.48), one can verify directly that, as $z \rightarrow 0$,

$$
\Upsilon(z)=\widehat{\Upsilon}(z) \begin{cases}z^{\frac{\alpha}{2} \sigma_{3}}\left(I+\frac{\sigma_{+}}{2 i \sin (\pi \alpha)}\right) \widehat{C}(z), & \text { for } \alpha \notin \mathbb{Z} \\ z^{\frac{\alpha}{2} \sigma_{3}}\left(I+\frac{(-1)^{\alpha}}{\pi i} \log \frac{\sqrt{z}}{2} \sigma_{+}\right) \widehat{C}(z), & \text { for } \alpha \in \mathbb{Z}\end{cases}
$$

where $\widehat{\Upsilon}(z)$ is an entire function and $\widehat{C}(z)$ is defined by

$$
\widehat{C}(z)=\left\{\begin{array}{cc}
I, & \arg z \in\left(-\frac{2}{3} \pi, \frac{2}{3} \pi\right), \\
\left(\begin{array}{cc}
1 & 0 \\
-e^{\alpha \pi i} & 1 \\
1 & 0 \\
e^{-\alpha \pi i} & 1
\end{array}\right), & \arg z \in\left(\frac{2}{3} \pi, \pi\right), \\
& \arg z \in\left(-\pi,-\frac{2}{3} \pi\right) .
\end{array}\right.
$$

\subsection{Local parametrix near the origin}

Near the origin, the outer parametrix $\Upsilon(z)$ is not a good approximation to $U(z)$ due to the singular behaviors of $U(z)$ at $z=0$ and $z=-\lambda^{2} s$. Thus, we need to construct a local parametrix $P_{0}$ in a neighborhood of the origin $D_{0}:=\{z:|z|<\epsilon\}$ with $\epsilon$ fixed but sufficiently small.

RH problem 7.3. We look for a $2 \times 2$ matrix-valued function $P_{0}(z)$ satisfying 
(1) $P_{0}(z)$ is defined and analytic in $\overline{D_{0}} \backslash\left\{\cup_{j=1}^{4} \Gamma_{j}^{\left(\lambda^{2} s\right)} \cup\left\{-\lambda^{2} s\right\}\right\}$.

(2) $P_{0}(z)$ satisfies the same jump conditions as $U$ on $D_{0} \cap\left\{\cup_{j=1}^{4} \Gamma_{j}^{\left(\lambda^{2} s\right)}\right\}$.

(3) $U(z) P_{0}(z)^{-1}$ is bounded at $z=0$ and $z=-\lambda^{2} s$.

(4) As $\lambda \rightarrow 0^{+}$, we have the matching condition

$$
P_{0}(z)=\left(I+O\left(\lambda^{2(1+\alpha)}\right)\right) \Upsilon(z)
$$

for $z \in \partial D_{0}$.

The construction of the local parametrix $P_{0}$ is similar to the ones in [3] and 44]. It is explicitly given by

$$
P_{0}(z)=\widehat{\Upsilon}(z)\left(I+(f(z ; \lambda)-h(z)) \sigma_{+}\right) e^{\frac{(-1)^{k+1} \lambda^{2 k}}{z^{k}} \sigma_{3}} z^{\frac{\alpha}{2} \sigma_{3}} \widetilde{C}(z),
$$

where $\widehat{\Upsilon}(z)$ is given in (7.10) and

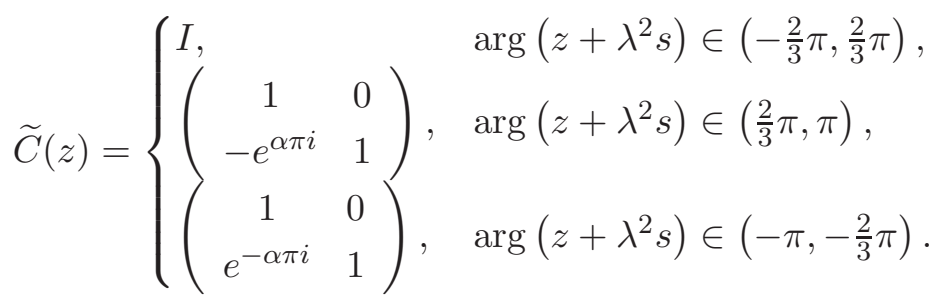

The scalar functions $f(z ; \lambda)$ and $h(z)$ in (7.13) are defined as follows:

$$
f(z ; \lambda)=\frac{e^{-z}}{2 \pi i} \int_{-\infty}^{-\lambda^{2} s} \frac{|x|^{\alpha} e^{x}}{x-z} e^{2 \frac{(-1)^{k+1} \lambda^{2 k}}{x^{k}}} d x
$$

and

$$
h(z)=f(z ; 0)- \begin{cases}\frac{z^{\alpha}}{2 i \sin (\pi \alpha)}, & \text { for } \alpha \notin \mathbb{Z}, \\ \frac{(-1)^{\alpha} z^{\alpha}}{\pi i} \log \frac{\sqrt{z}}{2}, & \text { for } \alpha \in \mathbb{Z} .\end{cases}
$$

It is straightforward to check that $h(z)$ is an entire function, $f(z ; \lambda)$ is analytic for $z \in \mathbb{C} \backslash$ $\left(-\infty,-\lambda^{2} s\right)$ and satisfies the jump condition

$$
f_{+}(x ; \lambda)-f_{-}(x ; \lambda)=|x|^{\alpha} e^{2 \frac{(-1)^{k+1} \lambda^{2 k}}{x^{k}}}, \quad x \in\left(-\infty,-\lambda^{2} s\right),
$$

with the following endpoint behavior

$$
f(z ; \lambda)=\frac{\left(\lambda^{2} s\right)^{\alpha} e^{-\frac{2}{s^{k}}}}{2 \pi i} \log \left(z+\lambda^{2} s\right)+O(1),
$$

as $z \rightarrow-\lambda^{2} s$. Moreover, uniformly for $z \in \partial D_{0}$, we have

$$
\begin{aligned}
f(z ; \lambda)-h(z)= & \frac{e^{-z}}{2 \pi i}\left[\int_{-\infty}^{0} \frac{|x|^{\alpha} e^{x}}{x-z}\left(e^{2 \frac{(-1)^{k+1} \lambda^{2 k}}{x^{k}}}-1\right) d x-\int_{-\lambda^{2} s}^{0} \frac{|x|^{\alpha} e^{x}}{x-z} e^{2 \frac{(-1)^{k+1} \lambda^{2 k}}{x^{k}}} d x\right] \\
& +\frac{z^{\alpha}}{2 i \sin (\pi \alpha)} \\
= & O\left(\lambda^{2 k}\right)+O\left(\lambda^{2(1+\alpha)}\right)+\frac{z^{\alpha}}{2 i \sin (\pi \alpha)} \\
= & \frac{z^{\alpha}}{2 i \sin (\pi \alpha)}+O\left(\lambda^{2(1+\alpha)}\right), \quad \text { as } \lambda \rightarrow 0^{+}, \text {if } \alpha \notin \mathbb{Z} .
\end{aligned}
$$


Similarly, if $\alpha \in \mathbb{Z}$, we also have

$$
f(z ; \lambda)-h(z)=\frac{(-1)^{\alpha} z^{\alpha}}{\pi i} \log \frac{\sqrt{z}}{2}+O\left(\lambda^{2(1+\alpha)}\right),
$$

as $\lambda \rightarrow 0^{+}$, uniformly for $z \in \partial D_{0}$.

Now let us verify $P_{0}(z)$ defined in (7.13) is indeed a solution to $\mathrm{RH}$ problem 7.3 , By (7.13) and (7.17), it is easily seen that $P_{0}$ satisfies the same jump conditions as $U$ on $D_{0} \cap\left\{\cup_{j=1}^{4} \Gamma_{j}^{\left(\lambda^{2} s\right)}\right\}$. Regarding item (3) in RH problem 7.3 , it follows from (7.4) and (7.13) that as $z \rightarrow 0$,

$$
U(z) P_{0}(z)^{-1}=O(1)\left(I-(f(z ; \lambda)-h(z)) \sigma_{+}\right) \widehat{\Upsilon}(z)^{-1}=O(1),
$$

since the functions $f, g$ and $\widehat{\Upsilon}$ are regular near the origin. Similarly, if $z \rightarrow-\lambda^{2} s$ and $\arg \left(z+\lambda^{2} s\right) \in\left(\frac{2 \pi}{3}, \pi\right)$, we have from (7.5) and (7.13) that

$$
\begin{aligned}
U(z) P_{0}(z)^{-1}= & O(1)\left(I+\frac{1}{2 \pi i} \log \left(z+\lambda^{2} s\right) \sigma_{+}\right)\left(\begin{array}{cc}
e^{\frac{\alpha \pi i}{2}} & 0 \\
-e^{\frac{\alpha \pi i}{2}} & e^{-\frac{\alpha \pi i}{2}}
\end{array}\right)\left(\begin{array}{cc}
1 & 0 \\
e^{\alpha \pi i} & 1
\end{array}\right) \\
& \times z^{-\frac{\alpha}{2} \sigma_{3}} e^{-\frac{(-1)^{k+1} \lambda^{2 k}}{z^{k}} \sigma_{3}}\left(I-(f(z ; \lambda)-h(z)) \sigma_{+}\right) \widehat{\Upsilon}(z)^{-1} \\
= & O(1)\left(I+\frac{z^{\alpha} e^{-\alpha \pi i}}{2 \pi i} e^{2 \frac{(-1)^{k+1} \lambda^{2 k}}{z^{k}}} \log \left(z+\lambda^{2} s\right) \sigma_{+}\right) \\
& \times\left(I-(f(z ; \lambda)-h(z)) \sigma_{+}\right) \widehat{\Upsilon}(z)^{-1} .
\end{aligned}
$$

Note that $h(z)$ and $\widehat{\Upsilon}(z)$ are entire functions and $\sigma_{+}^{2}=\mathbf{0}_{2 \times 2}$, using (7.18), we obtain

$$
U(z) P_{0}(z)^{-1}=O(1)
$$

as required. If $z$ approaches $-\lambda^{2} s$ from other regions, the above equation also holds by similar arguments.

Finally, to see the matching condition on $\partial D_{0}$, we observe from (7.10) and (7.13) that, if $\alpha \notin \mathbb{Z}$ and $\lambda \rightarrow 0^{+}$,

$$
P_{0}(z) \Upsilon(z)^{-1}=\widehat{\Upsilon}(z)\left(I+(f(z ; \lambda)-h(z)) \sigma_{+}\right) e^{\frac{(-1)^{k+1} \lambda^{2 k}}{z^{k}} \sigma_{3}}\left(I-\frac{z^{\alpha}}{2 i \sin (\pi \alpha)} \sigma_{+}\right) \widehat{\Upsilon}(z)^{-1} .
$$

It then follows from (7.19) that (7.12) holds for $\alpha \notin \mathbb{Z}$. Similarly, if $\alpha \in \mathbb{Z}$, the claim follows from (7.10) and (7.20).

\subsection{Final transformation}

The final transformation is defined by

$$
Q(z)= \begin{cases}U(z) \Upsilon(z)^{-1}, & \text { for } z \in \mathbb{C} \backslash D_{0}, \\ U(z) P_{0}(z)^{-1}, & \text { for } z \in D_{0} .\end{cases}
$$

Since the point $z=-\lambda^{2} s$ is located inside $D_{0}:=\{z:|z|<\epsilon\}$ when $\lambda$ is small enough, we may apply the contour deformation such that the jump contours of $U(z)$ and $\Upsilon(z)$ are the same outside $D_{0}$. It is then easily seen that $Q$ satisfies the following $\mathrm{RH}$ problem.

RH problem 7.4. The function $Q(z)$ defined in (7.22) has the following properties: 
(1) $Q(z)$ is analytic in $\mathbb{C} \backslash \partial D_{0}$.

(2) $Q(z)$ satisfies the jump condition

$$
Q_{+}(z)=Q_{-}(z) J_{Q}(z), \quad z \in \partial D_{0},
$$

where

$$
J_{Q}(z)=P_{0}(z) \Upsilon(z)^{-1} .
$$

(3) As $z \rightarrow \infty$,

$$
Q(z)=I+O(1 / z) .
$$

From the matching condition (7.12), we obtain

$$
J_{Q}(z)=I+O\left(\lambda^{2(1+\alpha)}\right), \quad \text { as } \lambda \rightarrow 0^{+} .
$$

By standard nonlinear steepest descent arguments (cf. [18, 22]), it then follows that

$$
Q(z)=I+O\left(\lambda^{2(1+\alpha)}\right), \quad \text { as } \lambda \rightarrow 0^{+},
$$

uniformly for $z$ in the complex plane off the jump contour.

Now we are ready to prove Theorems 2.4 and 2.5 .

\section{Proofs of Theorems 2.4 and 2.5}

\subsection{Proofs of Theorem 2.4 and (2.11)}

The existence of solutions to the coupled Painelvé system (2.4) and their analyticity for $\lambda>0$ follow directly from Theorem 6.3 and Proposition 6.4.

To see the asymptotics of $a(\lambda ; s)$ as $\lambda \rightarrow 0^{+}$, we note from (6.4) and (7.1) that

$$
a(\lambda ; s)=i \lambda^{-1}\left(U_{\infty}\right)_{12} .
$$

By (7.22), one has

$$
U(z)=Q(z) \Upsilon(z), \quad z \in \mathbb{C} \backslash D_{0} .
$$

The asymptotics of $a(\lambda ; s)$ given in (2.10) then follows from (7.9) and (77.25).

Finally, we show the asymptotics of $b_{1}(\lambda ; s)$ as $\lambda \rightarrow 0^{+}$. In view of (6.10) and (77.1), it is readily seen that

$$
b_{1}(\lambda ; s)=\frac{\lambda}{2}-\lambda i \lim _{z \rightarrow-\lambda^{2} s}\left(z+\lambda^{2} s\right)\left(U_{+}^{\prime}(z) U_{+}(z)^{-1}\right)_{12} .
$$

Since $\lambda \rightarrow 0^{+}, z=-\lambda^{2} s$ is close to the origin. For $z \in D_{0}$, we see from the definitions of $P_{0}(z)$ in (7.13) and $Q(z)$ in (7.22) that

$$
U(z)=Q(z) P_{0}(z)=Q(z) \widehat{\Upsilon}(z)\left(I+(f(z ; \lambda)-h(z)) \sigma_{+}\right) e^{\frac{(-1)^{k+1} \lambda^{2 k}}{z^{k}} \sigma_{3}} z^{\frac{\alpha}{2} \sigma_{3}},
$$

where $f(z ; \lambda)$ and $h(z)$ are defined in (7.15) and (7.16), respectively. By (8.3), we further have

$$
\begin{aligned}
U^{\prime}(z) U(z)^{-1}= & Q^{\prime}(z) Q(z)^{-1}+Q(z) \widehat{\Upsilon}^{\prime}(z) \widehat{\Upsilon}(z)^{-1} Q(z)^{-1} \\
& +Q(z) \widehat{\Upsilon}(z)\left(f^{\prime}(z ; \lambda)-h^{\prime}(z)\right) \sigma_{+}\left(I-(f(z ; \lambda)-h(z)) \sigma_{+}\right) \widehat{\Upsilon}(z)^{-1} Q(z)^{-1} \\
& +Q(z) \widehat{\Upsilon}(z)\left(I+(f(z ; \lambda)-h(z)) \sigma_{+}\right)\left(\frac{(-1)^{k} k \lambda^{2 k}}{z^{k+1}}+\frac{\alpha}{2 z}\right) \sigma_{3} \\
& \quad \times\left(I-(f(z ; \lambda)-h(z)) \sigma_{+}\right) \widehat{\Upsilon}(z)^{-1} Q(z)^{-1} .
\end{aligned}
$$


All functions on the right hand side of the above formula are analytic at $z=-\lambda^{2} s$ except $f(z ; \lambda)$; see (7.18). Therefore, as $z \rightarrow-\lambda^{2} s$, we obtain

$$
\begin{aligned}
\lim _{z \rightarrow-\lambda^{2} s}\left(z+\lambda^{2} s\right)\left(U_{+}^{\prime}(z) U_{+}(z)^{-1}\right)_{12} & =\lim _{z \rightarrow-\lambda^{2} s}\left(z+\lambda^{2} s\right) f^{\prime}(z ; \lambda)\left(Q(z) \widehat{\Upsilon}(z) \sigma_{+} \widehat{\Upsilon}(z)^{-1} Q(z)^{-1}\right)_{12} \\
& =\frac{\left(\lambda^{2} s\right)^{\alpha} e^{-\frac{2}{s^{k}}}}{2 \pi i}\left(Q\left(-\lambda^{2} s\right) \widehat{\Upsilon}\left(-\lambda^{2} s\right)\right)_{11}^{2}
\end{aligned}
$$

A combination of the above formula and (8.2) gives us

$$
b_{1}(\lambda ; s)=\frac{\lambda}{2}-\frac{s^{\alpha} \lambda^{2 \alpha+1}}{2 \pi} e^{-\frac{2}{s^{k}}}\left(Q\left(-\lambda^{2} s\right) \widehat{\Upsilon}\left(-\lambda^{2} s\right)\right)_{11}^{2} .
$$

From (7.8) and (7.10), the entire function $\widehat{\Upsilon}(z)$ takes the following form:

$$
\widehat{\Upsilon}(z)=\left(I+\frac{i}{8}\left(4 \alpha^{2}+3\right) \sigma_{-}\right) \pi^{\frac{\sigma_{3}}{2}} \Phi_{\mathrm{Bes}}^{(\alpha)}(z / 4) \widehat{C}(z)^{-1}\left(\begin{array}{cc}
1 & * \\
0 & 1
\end{array}\right) z^{-\frac{\alpha}{2} \sigma_{3}}
$$

Note that, in the above formula, the $(1,1)$ entry of $\pi^{\frac{\sigma_{3}}{2}} \Phi_{\mathrm{Bes}}^{(\alpha)}(z / 4) \widehat{C}(z)^{-1}$ remains unchanged when it is multiplied by a lower triangular matrix on its left or by an upper triangular matrix on its right. It then follows from (3.48) and (8.6) that

$$
(\widehat{\Upsilon}(z))_{11}=\left(\pi^{\frac{\sigma_{3}}{2}} \Phi_{\mathrm{Bes}}^{(\alpha)}(z / 4) \widehat{C}(z)^{-1} z^{-\frac{\alpha}{2} \sigma_{3}}\right)_{11}=\sqrt{\pi} z^{-\alpha / 2} I_{\alpha}\left(z^{1 / 2}\right)=\frac{\sqrt{\pi}}{2^{\alpha}} \sum_{k=0}^{\infty} \frac{(z / 4)^{k}}{k ! \Gamma(k+\alpha+1)}
$$

cf. [1]. Combining (7.25), (8.5) and the above formula, we obtain

$$
b_{1}(\lambda ; s)=\frac{\lambda}{2}-\frac{s^{\alpha} \lambda^{2 \alpha+1}}{2^{2 \alpha+1} \Gamma(\alpha+1)^{2}} e^{-\frac{2}{s^{k}}}\left(1+O\left(\lambda^{2(1+\alpha)}\right)\right),
$$

which is given in (2.11).

This completes the proofs of Theorem 2.4 and (2.11).

Remark 8.1. To prove Theorem 2.5, we need to know a little more about the properties of $M(z)$ as $z \rightarrow-s$ in (6.6) and (6.7). It is easily seen from (6.7) that $M_{1}^{(s)}=\lim _{z \rightarrow-s} M^{(s)}(z)^{-1} M^{(s)}(z)^{\prime}$. Then, it follows from (6.6), (6.8) and (7.1) that

$$
\left(M_{1}^{(s)}\right)_{21}=e^{\alpha \pi i} \lim _{z \rightarrow-s}\left(M_{-}^{-1}(z) M_{-}^{\prime}(z)\right)_{21}=e^{\alpha \pi i} \lambda^{2} \lim _{z \rightarrow-\lambda^{2} s}\left(U_{-}^{-1}(z) U_{-}^{\prime}(z)\right)_{21} .
$$

Using (8.3) again and conducting similar calculations in the above proofs, we have the following estimate

$$
\left(M_{1}^{(s)}\right)_{21}=O\left(\lambda^{2(1+\alpha)}\right), \quad \text { as } \lambda \rightarrow 0^{+} .
$$

\subsection{Proof of Theorem 2.5}

Recall the connection between $F_{s}\left(\lambda^{2} s ; \lambda^{2}\right)$ and $M$ given in (6.2), we then have from (8.8) that

$$
F_{s}\left(\lambda^{2} s ; \lambda^{2}\right)=-\frac{1}{2 \pi i \lambda^{2}}\left(M_{1}^{(s)}\right)_{21}, \quad \lambda>0 .
$$

Inserting (6.6) and (6.7) into (6.11), we obtain, on one hand,

$$
\frac{d}{d \lambda}\left(M_{1}^{(s)}\right)_{21}=\left(M_{0}^{(s)}\right)_{11}^{2}
$$


On the other hand, by (6.13) and (6.19), it follows that

$$
\left(M_{0}^{(s)}\right)_{11}^{2}=2 \pi i\left(\frac{\lambda}{2}-b_{1}(\lambda ; s)\right) .
$$

On account of the fact that (see (8.9))

$$
\lim _{\lambda \rightarrow 0^{+}}\left(M_{1}^{(s)}\right)_{21}=0,
$$

we obtain from (8.10) -8.12 that

$$
F_{s}\left(\lambda^{2} s ; \lambda^{2}\right)=-\frac{1}{\lambda^{2}} \int_{0}^{\lambda}\left(\frac{\tau}{2}-b_{1}(\tau ; s)\right) d \tau,
$$

where the integral is convergent by the asymptotics of $b_{1}(\lambda ; s)$ as $\lambda \rightarrow 0^{+}$given in (8.7). Equivalently, by (2.6), it follows that

$$
\frac{\partial}{\partial s} F\left(\lambda^{2} s ; \lambda^{2}\right)=\lambda^{2} F_{s}\left(\lambda^{2} s ; \lambda^{2}\right)=-\int_{0}^{\lambda} a_{s}(\tau ; s) d \tau .
$$

Since $F\left(0 ; \lambda^{2}\right)=0$, we integrate the above formula and arrive at (2.12).

This completes the proof of Theorem 2.5.

\section{A Consistency of (2.12) with the logarithm of (1.26)}

From (2.12), we have

$$
\frac{\partial}{\partial \lambda} F\left(\lambda^{2} s ; \lambda^{2}\right)=-(a(\lambda ; s)-a(\lambda ; 0)),
$$

where the function $a(\lambda ; s)$ is among the class of special solutions to the coupled Painleve III system (2.4) as stated in Theorem 2.4. This, together with the second equality in (4.6) and change of variables $\lambda^{2} s \rightarrow s$ and $\lambda^{2} \rightarrow \lambda$, implies that

$$
s F_{s}(s ; \lambda)=-\frac{1}{2} \sqrt{\lambda}(a(\sqrt{\lambda} ; s / \lambda)-a(\sqrt{\lambda} ; 0))-\lambda F_{\lambda}(s ; \lambda) .
$$

We next relate the limit $F_{s}(s ; \lambda)$ as $\lambda \rightarrow 0^{+}$to the $\mathrm{RH}$ problem for $X$ in Proposition 3.2 . From (4.9) and (4.52), we get

$$
F_{\lambda}\left(\lambda^{2} s ; \lambda^{2}\right)=\frac{r\left(\lambda^{2}\right)}{2 \lambda^{2}}+\frac{\alpha^{2}}{4 \lambda^{2}}-\frac{1}{16 \lambda^{2}}+O\left(s^{-1 / 2}\right), \quad \text { as } s \rightarrow+\infty .
$$

Due to the change of variables $\lambda^{2} s \rightarrow s$ and $\lambda^{2} \rightarrow \lambda$, the above formula and (1.22) gives us

$$
\lambda F_{\lambda}(s ; \lambda)=O\left(\lambda^{3 / 2}\right), \quad \text { as } \lambda \rightarrow 0^{+},
$$

uniformly for $s$ in any compact subset of $(0,+\infty)$. From the asymptotics of $a(\lambda ; s)$ as $\lambda \rightarrow 0^{+}$ given in (2.10), we also have

$$
\sqrt{\lambda} a(\sqrt{\lambda} ; 0)=\frac{1-4 \alpha^{2}}{8}+O\left(\lambda^{1+\alpha}\right) .
$$


Inserting the above two formulas into (A.2), we obtain

$$
F_{s}(s ; \lambda)=-\frac{1}{2 s}\left(\sqrt{\lambda} a(\sqrt{\lambda} ; s / \lambda)-\frac{1-4 \alpha^{2}}{8}\right)+o(1), \quad \text { as } \lambda \rightarrow 0^{+},
$$

where $o(1)$ term is uniform for $s$ in any compact subset of $(0,+\infty)$. On the other hand, on account of (6.1) and (6.4), we have

$$
\sqrt{\lambda} a(\sqrt{\lambda} ; s / \lambda)=i\left(X_{\infty}\right)_{12}(\lambda ; s)
$$

where $X_{\infty}$ is given in (3.16). A combination of (A.6) and (A.7) then gives us

$$
F_{s}(s ; \lambda)=-\frac{i}{2 s}\left(X_{\infty}\right)_{12}(\lambda ; s)+\frac{1-4 \alpha^{2}}{16 s}+o(1), \quad \text { as } \lambda \rightarrow 0^{+},
$$

uniformly for $s$ in any compact subset of $(0,+\infty)$.

Let us compare our RH problem for $X$ in Proposition 3.2 with $\mathrm{RH}$ problem 6.2 for the Bessel kernel in Bothner et al. [8]. They satisfy the following simple relation:

$$
X^{\mathrm{BIP}}(z ; s, v=0)=e^{\frac{\pi}{4} i \sigma_{3}} X(-s z ; s, \lambda=0),
$$

where $X^{\mathrm{BIP}}(z ; s, v)$ is the solution to RH problem 6.2 in [8]. Similar to (3.16), let us denote the $1 / z$ coefficient in the large $z$ expansion of $X^{\text {BIP }}$ by $X_{\infty}^{\text {BIP }}$. From [8, Equation (6.3)], the large $z$ expansions of RH problems 6.2 and B.1 in [8], we have

$$
\left(X_{\infty}^{\mathrm{BIP}}\right)_{12}=2 \mathcal{H}_{H}(\mathfrak{q}, \mathfrak{p}, s)+\frac{4 \alpha^{2}-1}{8 s}
$$

where

$$
\mathcal{H}_{H}(\mathfrak{q}, \mathfrak{p}, s)=\frac{\mathfrak{q}^{2}-1}{4 s} \mathfrak{p}^{2}-\frac{\alpha^{2} \mathfrak{q}^{2}}{4 s\left(\mathfrak{q}^{2}-1\right)}-\frac{\mathfrak{q}^{2}}{4},
$$

is the Hamiltonian for the Painlevé III equation; see [8, Equation (1.13)]. Here, $\mathfrak{q}(s)$ satisfies the equation (1.27) and the boundary condition (1.28), and $\mathfrak{p}(s)=2 s \mathfrak{q}^{\prime}(s) /\left(\mathfrak{q}^{2}(s)-1\right)$. Then, the formulas (A.8)-

$$
F_{s}(s ; \lambda)=\mathcal{H}_{H}(s)+o(1), \quad \text { as } \lambda \rightarrow 0^{+},
$$

uniformly for $s$ in any compact subset of $(0,+\infty)$.

From (1.11), (1.13), one can derive that

$$
K_{\mathrm{PIII}}(u, v ; \lambda)=O\left(e^{-c\left[\left(\frac{\lambda}{u}\right)^{k}+\left(\frac{\lambda}{u}\right)^{k}\right]}\right), \quad \text { as } u, v \rightarrow 0^{+},
$$

for $\lambda>0$ and certain constant $c>0$. Hence, $F_{\lambda}(s ; \lambda) / s$ is bounded when $s$ is small and positive. This, together with (A.2) and (A.12), implies that we can interchange the integral with respect to $s$ on both sides of (A.12) and the limit $\lambda \rightarrow 0^{+}$. Therefore, we obtain

$$
\lim _{\lambda \rightarrow 0^{+}} F(s ; \lambda)=\int_{0}^{s} \mathcal{H}_{H}(\tau) d \tau .
$$

Finally, due to the fact that $\left[-\tau \mathcal{H}_{H}(\tau)\right]^{\prime}=\mathfrak{q}^{2}(\tau) / 4$ (cf. [38, Equation (2.26)]), an integration by parts of the above formula gives us the logarithm of (1.26). 


\section{Acknowledgements}

We thank the anonymous referees for their careful reading and constructive suggestions. We also thank Prof. Yang Chen for drawing our attention to the preprint [32] upon completion of the present work. Dan Dai was partially supported by grants from the Research Grants Council of the Hong Kong Special Administrative Region, China (Project No. CityU 11300814, CityU 11300115, CityU 11303016). Shuai-Xia Xu was partially supported by National Natural Science Foundation of China under grant numbers 11571376 and 11201493, GuangDong Natural Science Foundation under grant number 2014A030313176. Lun Zhang was partially supported by National Natural Science Foundation of China under grant number 11501120, by The Program for Professor of Special Appointment (Eastern Scholar) at Shanghai Institutions of Higher Learning, and by Grant EZH1411513 from Fudan University.

\section{References}

[1] M. Abramowitz and I. A. Stegun, Handbook of mathematical functions with formulas, graphs, and mathematical tables, Dover, New York, 1972.

[2] G. Akemann, D. Villamaina and P. Vivo, A singular-potential random matrix model arising in mean-field glassy systems, Phys. Rev. E 89 (2014), 062146.

[3] M. Atkin, T. Claeys and F. Mezzadri, Random matrix ensembles with singularities and a hierarchy of Painlevé III equations, Int. Math. Res. Notices 2016 (2016), 2320-2375.

[4] M. V. Berry and P. Shukla, Tuck's incompressibility function: statistics for zeta zeros and eigenvalues, J. Phys. A 41 (2008), 385202.

[5] A. Borodin and P. Deift, Fredholm determinants, Jimbo-Miwa-Ueno $\tau$-functions, and representation theory, Comm. Pure Appl. Math. 55 (2002), 1160-1230.

[6] T. Bothner, From gap probabilities in random matrix theory to eigenvalue expansions, J. Phys. A 49 (2016), 075204.

[7] T. Bothner and A. Its, Asymptotics of a Fredholm determinant corresponding to the first bulk critical universality class in random matrix models, Comm. Math. Phys. 328 (2014), $155-202$.

[8] T. Bothner, A. Its and A. Prokhorov, On the analysis of incomplete spectra in random marix theory through an extension of the Jimbo-Miwa-Ueno differntial, preprint arXiv: 1708.06480 .

[9] L. Brightmore, F. Mezzadri and M. Y. Mo, A matrix model with a singular weight and Painlevé III, Comm. Math. Phys. 333 (2015), 1317-1364.

[10] P. W. Brouwer, K. M. Frahm and C. W. Beenakker, Distribution of the quantum mechanical time-delay matrix for a chaotic cavity, Waves Random Media 9 (1999), 91-104.

[11] P. W. Brouwer, K. M. Frahm and C. W. Beenakker, Quantum mechanical time-delay matrix in chaotic scattering, Phys. Rev. Lett. 78 (1997), 4737-4740.

[12] C. Charlier and A. Doeraene, The generating function for the Bessel point process and a system of coupled Painlevé V equations, preprint arXiv:1709.07365. 
[13] Y. Chen, K. Eriksen and C. A. Tracy, Largest eigenvalue distribution in the double scaling limit of matrix models: a Coulomb fluid approach, J. Phys. A 28 (1995), L207-L211.

[14] Y. Chen and A. Its, Painlevé III and a singular linear statistics in Hermitian random matrix ensembles I, J. Approx. Theory 162 (2010), 270-297.

[15] T. Claeys and A. Doeraene, The generating function for the Airy point process and a system of coupled Painlevé II equations, to appear in Stud. Appl. Math., preprint arXiv:1708.03481.

[16] T. Claeys, M. Girotti and D. Stivigny, Large gap asymptotics at the hard edge for product random matrices and Muttalib-Borodin ensembles, to appear in Int. Math. Res. Notices, preprint arXiv:1612.01916.

[17] T. Claeys, A. Its and I. Krasovsky, Higher-order analogues of the Tracy-Widom distribution and the Painlevé II hierarchy, Comm. Pure Appl. Math. 63 (2010), 362-412.

[18] P. Deift, Orthogonal polynomials and random matrices: a Riemann-Hilbert approach, vol. 3 of Courant Lecture Notes in Mathematics, New York University Courant Institute of Mathematical Sciences, New York, 1999.

[19] P. Deift, A. Its and X. Zhou, A Riemann-Hilbert approach to asymptotic problems arising in the theory of random matrix models, and also in the theory of integrable statistical mechanics, Ann. of Math. (2) 146 (1997), 149-235.

[20] P. Deift, I. Krasovsky and J. Vasilevska, Asymptotics for a determinant with a confluent hypergeometric kernel, Int. Math. Res. Notices 2011 (2011), 2117-2160.

[21] P. Deift, T. Kriecherbauer, K. T.-R. McLaughlin, S. Venakides and X. Zhou, Uniform asymptotics for polynomials orthogonal with respect to varying exponential weights and applications to universality questions in random matrix theory, Comm. Pure Appl. Math. 52 (1999), no. 11, 1335-1425.

[22] P. Deift and X. Zhou, A steepest descent method for oscillatory Riemann-Hilbert problems. Asymptotics for the MKdV equation, Ann. of Math. (2) 137 (1993), 295-368.

[23] F. Dyson, Fredholm determinants and inverse scattering problems, Commun. Math. Phys. 47 (1976), 171-183.

[24] A. S. Fokas and X. Zhou, On the solvability of Painlevé II and IV, Comm. Math. Phys. 144 (1992), 601-622.

[25] P. J. Forrester, The spectrum edge of random matrix ensembles, Nucl. Phys. B 402 (1993), 709-728.

[26] M. Girotti, Gap probabilities for the generalized Bessel process: a Riemann-Hilbert approach, Math. Phys. Anal. Geom. 17 (2014), 183-211.

[27] A. R. Its, A. G. Izergin, V. E. Korepin and N. A. Slavnov, Differential equations for quantum correlation functions, Internat. J. Modern Phys. B 4 (1990), 1003-1037.

[28] A. R. Its, A. B. J. Kuijlaars and J. Östensson, Asymptotics for a special solution of the thirty fourth Painlevé equation, Nonlinearity 22 (2009), 1523-1558. 
[29] K. Johansson, Random matrices and determinantal processes, Mathematical statistical physics (Lecture notes of the Les Houches Summer School), Elsevier B. V., Amsterdam 2006, 1-55.

[30] A. B. J. Kuijlaars and K. T-R. McLaughlin, Generic behavior of the density of states in random matrix theory and equilibrium problems in the presence of real analytic external fields, Comm. Pure Appl. Math. 53 (2000), 736-785.

[31] A. B. J. Kuijlaars, K. T-R. McLaughlin, W. Van Assche and M. Vanlessen, The RiemannHilbert approach to strong asymptotics for orthogonal polynomials on $[-1,1]$, Adv. Math. 188 (2004), 337-398.

[32] S. Lyu, J. Griffin and Y. Chen, Hankel determinant generated by a singularly perturbed Laguerre weight on $[s, \infty)$, preprint (2017).

[33] F. W. J. Olver, D. W. Lozier, R. F. Boisvert and C. W. Clark, editors, NIST Handbook of Mathematical Functions, Cambridge University Press, Cambridge 2010. Print companion to $[\mathrm{DLMF}]$.

[34] A. Soshnikov, Determinantal random point fields, Russian Math. Surveys 55 (2000), 923975 .

[35] E. Strahov, Differential equations for singular values of products of Ginibre random matrices, J. Phys. A 47 (2014), 325203.

[36] C. Texier and S. N. Majumdar, Wigner time-delay distribution in chaotic cavities and freezing transition, Phys. Rev. Lett. 110 (2013), 250602.

[37] C. A. Tracy and H. Widom, Fredholm determinants, differential equations and matrix models, Comm. Math. Phys. 163 (1994), 33-72.

[38] C. A. Tracy and H. Widom, Level spacing distributions and the Bessel kernel, Comm. Math. Phys. 161 (1994), 289-309.

[39] C. A. Tracy and H. Widom, Level-spacing distributions and the Airy kernel, Comm. Math. Phys. 159 (1994), 151-174.

[40] C. A. Tracy and H. Widom, Introduction to random matrices. Geometric and quantum aspects of integrable systems (Scheveningen, 1992), Lecture Notes in Physics, vol 424, Berlin: Springer, 1993.

[41] M. Vanlessen, Strong asymptotics of Laguerre-type orthogonal polynomials and applications in random matrix theory, Constr. Approx. 25 (2007), 125-175.

[42] S.-X. Xu and D. Dai, Tracy-Widom distributions in critical unitary random matrix ensembles and the coupled Painlevé II system, preprint arXiv:1708.06113.

[43] S.-X. Xu, D. Dai and Y.-Q. Zhao, Painlevé III asymptotics of Hankel determinants for a singularly perturbed Laguerre weight, J. Approx. Theory 192 (2015), 1-18.

[44] S.-X. Xu, D. Dai and Y.-Q. Zhao, Critical edge behavior and the Bessel to Airy transition in the singularly perturbed Laguerre unitary ensemble, Comm. Math. Phys. 332 (2014), $1257-1296$.

[45] L. Zhang, On Wright's generalized Bessel kernel, Physica D 340 (2017), 27-39. 
[46] X. Zhou, The Riemann-Hilbert problem and inverse scattering, SIAM J. Math. Anal. 20 (1989), 966-986. 